TRANSACTIONS OF THE

AMERICAN MATHEMATICAL SOCIETY

Volume 354, Number 12, Pages 4847-4869

S 0002-9947(02)03109-4

Article electronically published on August 1, 2002

\title{
FOURIER EXPANSION OF EISENSTEIN SERIES ON THE HILBERT MODULAR GROUP AND HILBERT CLASS FIELDS
}

\author{
CLAUS MAZANTI SORENSEN
}

\begin{abstract}
In this paper we consider the Eisenstein series for the Hilbert modular group of a general number field. We compute the Fourier expansion at each cusp explicitly. The Fourier coefficients are given in terms of completed partial Hecke $L$-series, and from their functional equations, we get the functional equation for the Eisenstein vector. That is, we identify the scattering matrix. When we compute the determinant of the scattering matrix in the principal case, the Dedekind $\xi$-function of the Hilbert class field shows up. A proof in the imaginary quadratic case was given in Efrat and Sarnak, and for totally real fields with class number one a proof was given in Efrat.
\end{abstract}

\section{INTRODUCTION}

Let us consider the group $G=\mathrm{SL}(2, \mathbb{R})$. It acts transitively on the upper halfplane

$$
X=H_{2}=\{z=x+i y \in \mathbb{C}: y>0\}
$$

by linear fractional transformations. Thus for a $g \in G$ and a $z \in X$, we have

$$
g z=\frac{a z+b}{c z+d} \quad \text { where } g=\left(\begin{array}{ll}
a & b \\
c & d
\end{array}\right) \in G .
$$

The stabilizer at the point $z=i$ is $\mathrm{SO}(2)$, and hence there is a homeomorphism

$$
H_{2} \approx \mathrm{SL}(2, \mathbb{R}) / \mathrm{SO}(2) \text {. }
$$

We equip $\mathrm{H}_{2}$ with the Poincaré metric, so as to get a Riemannian manifold:

$$
d s(z)^{2}=\frac{d x^{2}+d y^{2}}{y^{2}} .
$$

Then $G$ acts by isometries. The associated $G$-invariant measure on $H_{2}$ is given by

$$
d \mu(z)=\frac{d x d y}{y^{2}} .
$$

The theory of automorphic forms emerged from the study of functions on $H_{2}$ that are invariant/automorphic under some discrete subgroup of $G=\operatorname{SL}(2, \mathbb{R})$. The first such that comes to mind is the full elliptic modular group

$$
\Gamma=\operatorname{SL}(2, \mathbb{Z})=\left\{\gamma=\left(\begin{array}{ll}
a & b \\
c & d
\end{array}\right): a, b, c, d \in \mathbb{Z} \text { and } a d-b c=1\right\} .
$$

Received by the editors March 26, 2002 and, in revised form, May 13, 2002.

2000 Mathematics Subject Classification. Primary 11F30, 11F41, 11M36, 11R37, 11R42.

Key words and phrases. Eisenstein series, Hilbert modular groups, Hilbert class fields. 
The group $\Gamma$ is not too small to be of interest. Any fundamental domain has finite measure. A concrete fundamental domain for $\Gamma$ is the famous

$$
F=\left\{z \in H_{2}:-\frac{1}{2}<\operatorname{Re}(z)<+\frac{1}{2} \text { and }|z|>1\right\} .
$$

By the Gauss-Bonnet theorem, $\mu(F)=\pi / 3$. As one can see, $F$ is not compact. It has a geodesic ray that stretches out to $\infty$. This is called a cusp. The fact that there is only one such cusp is, in fact, equivalent to the fundamental theorem of arithmetic pertaining to unique factorization of integers. The space of automorphic functions that we are interested in is

$$
L^{2}(\Gamma \backslash X)=\text { square integrable } \Gamma \text {-automorphic functions. }
$$

From the metric $d s(z)$ on $H_{2}$, we have a corresponding invariant Laplacian

$$
\Delta=y^{2}\left(\frac{\partial^{2}}{\partial x^{2}}+\frac{\partial^{2}}{\partial y^{2}}\right) .
$$

It acts on a dense subspace of $L^{2}(\Gamma \backslash X)$ and admits a unique positive selfadjoint extension $L=-\bar{\Delta}$. This differential operator has a continuous spectrum, and in order to determine it completely one introduces the Eisenstein series

$$
E(z, s)=\sum_{\gamma \in \Gamma_{\infty} \backslash \Gamma} y(\gamma z)^{s}
$$

absolutely convergent for $\operatorname{Re}(s)>1$. This is not square integrable, but it defines a $\Gamma$-automorphic generalized eigenfunction for $L$ :

$$
L E(z, s)=s(1-s) E(z, s)
$$

Now, $s(1-s)$ is a nonnegative real number if and only if either $s$ is real and $0 \leq s \leq 1$ or $\operatorname{Re}(s)=1 / 2$. Therefore, we need to show that $E(z, s)$ has meromorphic continuation to this region. In our case, where $\Gamma=\operatorname{SL}(2, \mathbb{Z})$, this continuation is accomplished by the Fourier expansion of $E(z, s)$. Clearly, for each $y>0$, the Eisenstein series is invariant under $x \mapsto x+1$ and hence has a Fourier expansion

$$
E(z, s)=\sum_{l \in \mathbb{Z}} a_{l}(y ; s) e^{2 \pi i l x}
$$

Using $L E(z, s)=s(1-s) E(z, s)$, we find that the $a_{l}(y ; s)$ satisfy the Bessel equation

$$
a_{l}^{\prime \prime}(y ; s)=\left\{4 \pi^{2} l^{2}-\frac{s(1-s)}{y^{2}}\right\} a_{l}(y ; s) .
$$

When $l=0$, the space of solutions is spanned by $y^{s}$ and $y^{1-s}$. Therefore, by looking at the leading term of $E(z, s)$, we find that for some $\Phi(s)$,

$$
a_{0}(y ; s)=y^{s}+\Phi(s) y^{1-s} \text {. }
$$

This $\Phi(s)$ is called the scattering matrix/coefficient. When $l \neq 0$, the Bessel equation has two independent solutions, one that increases asymptotically as $e^{2 \pi|l| y}$ as $y \rightarrow \infty$, and one that decays as $e^{-2 \pi|l| y}$ as $y \rightarrow \infty$. Only the decaying solution can appear in $a_{l}(y ; s)$ by the polynomial growth of the Eisenstein series. This is $\sqrt{y} K_{s-1 / 2}(2 \pi|l| y)$, where $K_{s}(y)$ is Macdonald's Bessel function

$$
K_{s}(y)=\frac{1}{2} \int_{0}^{\infty} \exp \left\{-\frac{1}{2} y\left(t+\frac{1}{t}\right)\right\} t^{s} \frac{d t}{t},
$$

defined for $y>0$ and all $s \in \mathbb{C}$. Therefore, when $l \neq 0$ for some $a_{l}(s)$, we have

$$
a_{l}(y ; s)=a_{l}(s) \sqrt{y} K_{s-1 / 2}(2 \pi|l| y) \text {. }
$$


Using the integral representations of $a_{l}(y ; s)$ as Fourier coefficients, one can justify the above, and find $\Phi(s)$ and $a_{l}(s)$ explicitly. One gets

$$
\Phi(s)=\frac{\xi(2 s-1)}{\xi(2 s)},
$$

where $\xi(s)=\pi^{-s / 2} \Gamma(s / 2) \zeta(s)$ is the completed Riemann zeta function. It satisfies the functional equation

$$
\xi(1-s)=\xi(s)
$$

Therefore, $\Phi(s)$ satisfies $\Phi(s) \Phi(1-s)=1$. One also finds the expression for $a_{l}(s)$ :

$$
a_{l}(s)=\frac{2|l|^{s-1 / 2} \sigma_{1-2 s}(l)}{\xi(2 s)},
$$

where $\sigma_{s}(l)=\sum_{d \mid l} d^{s}$ is the sum of divisor functions. Using that $\xi(s)$ has analytic continuation, we can now use the Fourier expansion to continue $E(z, s)$ to a meromorphic function on the whole $s$-plane. Furthermore, using the easy facts that $K_{s}(y)$ and $|l|^{-s / 2} \sigma_{s}(l)$ are both invariant under $s \mapsto-s$, we find that $E(z, s)$ satisfies a functional equation

$$
E(z, s)=\Phi(s) E(z, 1-s) .
$$

In this paper, we generalize the above to an arbitrary number field $K$. Let $r_{1}$ be the number of real places and let $r_{2}$ be the number of complex places. There is an associated symmetric space

$$
X=H_{2}^{r_{1}} \times H_{3}^{r_{2}}
$$

on which $G=\mathrm{SL}(2, \mathbb{R})^{r_{1}} \times \mathrm{SL}(2, \mathbb{C})^{r_{2}}$ acts transitively. We consider $\mathrm{SL}(2, O)$ as a discrete cofinite irreducible subgroup $\Gamma$ of isometries, by applying the complex embeddings of $K$. This is the Hilbert modular group. The action of $G$ extends to an action on

$$
\mathbb{P}=\mathbb{P}^{1}(\mathbb{R})^{r_{1}} \times \mathbb{P}^{1}(\mathbb{C})^{r_{2}}
$$

The cusps are, by definition, the parabolic fixpoints in $\mathbb{P}$. One can easily check that these are exactly the image of the natural map $\mathbb{P}^{1}(K) \rightarrow \mathbb{P}$. There are only finitely many inequivalent cusps. In fact, there is a canonical bijection

$$
\Gamma \backslash\{\text { cusps }\} \approx \mathrm{Cl},
$$

denoted by $\kappa \mapsto C_{\kappa}$. For each cusp $\kappa \in \mathbb{P}$ we have an Eisenstein series. To define them we choose a matrix $\sigma \in \operatorname{SL}(2, K) \subset G$ such that $\sigma \infty=\kappa$ and

$$
\sigma^{-1} \Gamma_{\kappa} \sigma=\left\{\left(\begin{array}{cc}
u & b \\
0 & u^{-1}
\end{array}\right): u \in O^{\times} \text {and } b \in \mathfrak{a}^{-2}\right\},
$$

for some integral ideal $\mathfrak{a} \in C_{\kappa}$. Also, we fix an ordered basis for $O^{\times}$. Then for each $m \in \mathbb{Z}^{\nu}$, where $\nu=\operatorname{rk} O^{\times}$, we have a character

$$
\chi_{m}:\left(\mathbb{R}^{*}\right)^{r_{1}} \times\left(\mathbb{C}^{*}\right)^{r_{2}} \rightarrow S^{1}
$$

that is trivial on $O^{\times}$. Hence it defines a character on the group of principal ideals. Now, for $z \in X, s \in \mathbb{C}, m \in \mathbb{Z}^{\nu}$ and a Grössencharakter $\chi$ modulo $\mathfrak{m}=O$ extending $\chi_{m}$, the Eisenstein series $E_{\kappa}(z, s, m, \chi)$ is defined as

$$
E_{\kappa}(z, s, m, \chi)=\frac{\mathrm{N}_{K / \mathbb{Q}}(\mathfrak{a})^{2 s}}{\chi(\mathfrak{a})} \sum_{\gamma \in \Gamma_{\kappa} \backslash \Gamma} \prod_{i=1}^{n} y_{i}\left(\sigma^{-1} \gamma z\right)^{s_{i}}
$$


where $s_{i}=s_{i}(s, m)$ are given explicitly from the basis for $O^{\times}$. The definition is independent of the choice of $\sigma$. The Eisenstein series converges absolutely for $\operatorname{Re}(s)$ sufficiently large. We want to study the asymptotics of $E_{\kappa}(z, s, m, \chi)$ as $z \rightarrow \kappa^{\prime}$. We do this by choosing a $\sigma^{\prime}$ as above and look at $E_{\kappa}\left(\sigma^{\prime} z, s, m, \chi\right)$ as $z \rightarrow \infty$. Only the dependence of $y=\left(y_{1}, \ldots, y_{n}\right) \in \mathbb{R}_{+}^{n}$ is important, since $x=\left(x_{1}, \ldots, x_{n}\right) \in$ $\mathbb{R}^{r_{1}} \times \mathbb{C}^{r_{2}}$ is bounded within a fundamental domain. Thus we fix a $y \in \mathbb{R}_{+}^{n}$, and look at $E_{\kappa}\left(\sigma^{\prime} z, s, m, \chi\right)$ as a function of $x \in \mathbb{R}^{r_{1}} \times \mathbb{C}^{r_{2}}$. Since Eisenstein series are automorphic, we find that it is $\mathfrak{b}^{\prime}$-periodic where $\mathfrak{b}^{\prime}=\mathfrak{a}^{\prime-2}$. Hence it has a Fourier expansion

$$
E_{\kappa}\left(\sigma^{\prime} z, s, m, \chi\right)=\sum_{l \in \mathfrak{b}^{\prime *}} a_{l}^{\kappa, \kappa^{\prime}}(y, s, m, \chi) e^{2 \pi i \operatorname{Tr}_{K / \mathbb{Q}}(l x)},
$$

where $\mathfrak{b}^{*}=\left\{l \in K: \operatorname{Tr}_{K / \mathbb{Q}}\left(l \mathfrak{b}^{\prime}\right) \subset \mathbb{Z}\right\}$ is the dual module and

$$
\operatorname{Tr}_{K / \mathbb{Q}}(x)=\sum_{i \leq r_{1}} x_{i}+\sum_{i>r_{1}} 2 \operatorname{Re}\left(x_{i}\right)
$$

for $x=\left(x_{1}, \ldots, x_{n}\right) \in \mathbb{R}^{r_{1}} \times \mathbb{C}^{r_{2}}$. The Fourier coefficients are given by

$$
a_{l}^{\kappa, \kappa^{\prime}}(y, s, m, \chi)=\frac{2^{r_{2}}}{\mathrm{~N}_{K / \mathbb{Q}}\left(\mathfrak{b}^{\prime}\right) \sqrt{|D|}} \int_{F_{\mathfrak{b}^{\prime}}} E_{\kappa}\left(\sigma^{\prime} z, s, m, \chi\right) e^{-2 \pi i \operatorname{Tr}_{K / \mathbb{Q}}(l x)} d x
$$

where $F_{\mathfrak{b}^{\prime}}$ is a fundamental mesh for the lattice, $D$ is the discriminant of $K$, and $d x$ is the usual Lebesgue measure on $\mathbb{R}^{r_{1}} \times \mathbb{C}^{r_{2}}$. We use this notation, keeping in mind that $a_{l}^{\kappa, \kappa^{\prime}}(y, s, m, \chi)$ depends on the choice of a scaling matrix $\sigma^{\prime}$. In this paper we compute these. To state the results, we introduce some notation. Let us recall the definition of the partial Hecke $L$-series $L(s, \chi, C)$, where $\chi: I \rightarrow S^{1}$ is a Grössencharakter modulo $\mathfrak{m}=O$ extending $\chi_{m}$, and $C \in \mathrm{Cl}$ is an ideal class:

$$
L(s, \chi, C)=\sum_{\substack{\mathfrak{a} \in C \\ \text { integral }}} \frac{\chi(\mathfrak{a})}{\mathrm{N}_{K / \mathbb{Q}}(\mathfrak{a})^{s}}
$$

The series is absolutely convergent for $\operatorname{Re}(s)>1$, and uniformly in $\operatorname{Re}(s)>1+\epsilon$ for every $\epsilon>0$. Thus $L(s, \chi, C)$ defines an analytic function for $\operatorname{Re}(s)>1$. We define the completed partial $L$-series by

$$
\Lambda(2 s, \chi, C)=2^{r_{2}+\left(s_{1}+\cdots+s_{r_{1}}\right)}(2 \pi)^{-d s}|D|^{s}\left\{\prod_{i=1}^{n} \Gamma\left(s_{i}\right)\right\} L(2 s, \chi, C),
$$

where $s_{i}=s_{i}(s, m)$. It is shown in [5] that $\Lambda(s, \chi, C)$ has a meromorphic continuation to the whole complex plane, and a functional equation

$$
\Lambda(s, \chi, C)=\chi(\mathfrak{d}) \Lambda\left(1-s, \bar{\chi}, C^{\prime}\right),
$$

where $C C^{\prime}=[\mathfrak{d}]$ is the class of the different $\mathfrak{d}$. Recall that by definition, $\mathfrak{d}^{-1}=O^{*}$. We put an order on the set of cusps and arrange the $\Lambda\left(s, \chi, C_{\kappa}^{-1} C_{\kappa^{\prime}}\right)$ in a matrix

$$
\hat{\Lambda}(s, \chi)=\left\{\Lambda\left(s, \chi, C_{\kappa}^{-1} C_{\kappa^{\prime}}\right)\right\}
$$


Then the constant term $a_{0}^{\kappa, \kappa^{\prime}}(y, s, m, \chi)$ is the $\kappa^{\prime}$-th entry of the column vector

$$
\begin{aligned}
a_{0}^{\kappa^{\prime}}(y, s, m, \chi)=e^{\kappa^{\prime}} \frac{\mathrm{N}_{K / \mathbb{Q}}\left(\mathfrak{a}^{\prime}\right)^{s}}{\chi\left(\mathfrak{a}^{\prime}\right)} y_{1}^{s_{1}} & \cdots y_{n}^{s_{n}} \\
& +\frac{\chi\left(\mathfrak{a}^{\prime}\right)}{\mathrm{N}_{K / \mathbb{Q}}\left(\mathfrak{a}^{\prime}\right)^{2 s-2}} \Phi^{\kappa^{\prime}}(s, m, \chi) y_{1}^{1-s_{1}} \cdots y_{n}^{2-s_{n}},
\end{aligned}
$$

where $\Phi^{\kappa^{\prime}}(s, m, \chi)$ is the $\kappa^{\prime}$-th column of

$$
\Phi(s, m, \chi)=\hat{\Lambda}(2 s, \chi)^{-1} \hat{\Lambda}(2 s-1, \chi) \mathbb{P},
$$

where $\mathbb{P}$ denotes the permutation matrix corresponding to inversion on $\mathrm{Cl}$. For nonzero $l \in \mathfrak{b}^{\prime *}$, the Fourier coefficient $a_{l}^{\kappa, \kappa^{\prime}}(y, s, m, \chi)$ is the $\kappa^{\prime}$-th entry of the column vector

$$
a_{l}^{\kappa^{\prime}}(y, s, m, \chi)=\frac{\chi\left(\mathfrak{a}^{\prime}\right)}{\mathrm{N}_{K / \mathbb{Q}}\left(\mathfrak{a}^{\prime}\right)^{2 s-2}} a_{l}^{\kappa^{\prime}}(s, m, \chi) \sqrt{y_{1} \cdots y_{n}^{2}} K_{s-1 / 2}(y, l, m),
$$

where

$$
a_{l}^{\kappa^{\prime}}(s, m, \chi)=2^{d} \mathrm{~N}_{K / \mathbb{Q}}(\mathfrak{d} l)^{s-1 / 2} \chi_{m / 2}(l)^{-1} \hat{\Lambda}(2 s, \chi)^{-1} \sigma_{1-2 s}^{\kappa^{\prime}}(\chi, l) .
$$

Here $\sigma_{s}^{\kappa^{\prime}}(\chi, l)$ is a column vector whose entries are analogous to the sum of divisor functions, and $K_{s}(y, l, m)$ is a product of Macdonald's Bessel functions

$$
K_{s}(y, l, m)=K_{s_{1}}\left(2 \pi\left|l^{(1)}\right| y_{1}\right) \cdots K_{s_{n}}\left(4 \pi\left|l^{(n)}\right| y_{n}\right) .
$$

This gives us the analytic continuation of the Eisenstein series. In addition, from the functional equation for partial $L$-series, we get that

$$
\mathfrak{E}(z, s, m, \chi)=\Phi(s, m, \chi) \mathfrak{E}(z, 1-s,-m, \bar{\chi}),
$$

where $\mathfrak{E}(z, s, m, \chi)$ is the Eisenstein vector with entries $E_{\kappa}(z, s, m, \chi)$. Therefore, $\Phi(s, m, \chi)$ is the scattering matrix for the Hilbert modular group. If we conjugate it by the character table of $\mathrm{Cl}$, we get a permuted diagonal matrix. Then it is easy to see that

$$
\operatorname{det} \Phi(s, m, \chi)=\operatorname{sign}(\mathrm{Cl}) \prod_{\psi} \frac{\Lambda(2 s-1, \psi)}{\Lambda(2 s, \psi)},
$$

where $\psi$ varies over all Grössencharakters modulo $\mathfrak{m}=O$ extending $\chi_{m}$ and $\operatorname{sign}(\mathrm{Cl})$ denotes the sign of the inversion map on $\mathrm{Cl}$. For $m=0$ we use class field theory and the Artin factorization formula to see that

$$
\operatorname{det} \Phi(s, 0, \chi)=\operatorname{sign}(\mathrm{Cl}) \frac{\xi_{H}(2 s-1)}{\xi_{H}(2 s)},
$$

where $\xi_{H}(s)$ is the Dedekind $\xi$-function of the Hilbert class field $H$. In particular,

$$
\operatorname{det} \Phi(1 / 2,0, \chi)=-\operatorname{sign}(\mathrm{Cl}) .
$$

We also compute the trace of $\Phi(s, 0, \chi)$. It is given by

$$
\operatorname{tr} \Phi(s, m, \chi)=\sum_{\psi \text { real }} \frac{\Lambda(2 s-1, \psi \otimes \chi)}{\Lambda(2 s, \psi \otimes \chi)},
$$

where $\psi$ varies over all real characters of $\mathrm{Cl}$. For $s=1 / 2$, we see that

$$
\operatorname{tr} \Phi(1 / 2,0, \chi)=h[2]-2,
$$


using the analytic properties of $\Lambda(s, \psi)$ at $s=0$, the orthogonality relations for $\mathrm{Cl} / \mathrm{Cl}^{2}$ and the result of Hecke that $[\mathfrak{d}] \in \mathrm{Cl}^{2}$.

\section{The HilberT MOdular group AND its Cusps}

Let us recall a few basic facts about hyperbolic 2-space $H_{2}$ and hyperbolic 3space $H_{3}$. We always think of $H_{2}$ as the upper half-plane of $\mathbb{C}$. Points of $H_{2}$ are denoted by $z=x+i y$, and we equip $H_{2}$ with the Poincaré metric

$$
d s(z)^{2}=\frac{d x^{2}+d y^{2}}{y^{2}} .
$$

The group $\operatorname{SL}(2, \mathbb{R})$ acts transitively on $\mathrm{H}_{2}$ by linear fractional transformations, and these are isometries. The isotropy group at $i$ is $\mathrm{SO}(2)$, and we obtain a homeomorphism

$$
H_{2} \approx \mathrm{SL}(2, \mathbb{R}) / \mathrm{SO}(2) .
$$

From the metric on $\mathrm{H}_{2}$ we obtain a measure that is invariant under the action of $\mathrm{SL}(2, \mathbb{R})$. It is given by

$$
d \mu(z)=\frac{d x d y}{y^{2}} .
$$

Similarly, we always think of $H_{3}$ as the upper half-space $\mathbb{C} \times(0, \infty)$ inside the quaternions. Points of $H_{3}$ are denoted by $z=x+j y$, and we put the following metric on $H_{3}$ :

$$
d s(z)^{2}=\frac{d|x|^{2}+d y^{2}}{y^{2}} .
$$

The group $\mathrm{SL}(2, \mathbb{C})$ acts transitively on $H_{3}$ by linear fractional transformations, and these are isometries. The isotropy group at $j$ is $\mathrm{SU}(2)$, and we obtain a homeomorphism

$$
H_{3} \approx \mathrm{SL}(2, \mathbb{C}) / \mathrm{SU}(2) .
$$

As before, we obtain a measure $d \mu(z)$ on $H_{3}$ that is invariant under the action of $\mathrm{SL}(2, \mathbb{C})$. It is given by

$$
d \mu(z)=\frac{d x d y}{y^{3}},
$$

where $d x$ is the usual Lebesgue measure on $\mathbb{C}$. In this paper, we consider the space

$$
X=H_{2}^{r_{1}} \times H_{3}^{r_{2}} .
$$

Points of $X$ are denoted by $z=\left(z_{1}, \ldots, z_{n}\right)$, where $n=r_{1}+r_{2}$. As a product of Riemannian manifolds, the space $X$ has a metric $d s(z)^{2}$ and a corresponding measure $d \mu(z)$. The group

$$
G=\mathrm{SL}(2, \mathbb{R})^{r_{1}} \times \mathrm{SL}(2, \mathbb{C})^{r_{2}}
$$

acts transitively on $X$ by isometries. The isotropy group at the point $(i, \ldots, j)$ is the maximal compact subgroup $\mathrm{SO}(2)^{r_{1}} \times \mathrm{SU}(2)^{r_{2}}$, and we obtain a homeomorphism between $X$ and the quotient of $G$ by this subgroup. Throughout this paper we fix a number field $K$ with $r_{1}$ real primes and $r_{2}$ complex primes. Hence the degree is

$$
d=(K: \mathbb{Q})=r_{1}+2 r_{2} .
$$

The infinite primes are represented by a certain set of embeddings $K \rightarrow \mathbb{C}$ that we also fix. In addition, we put a total order on this set, such that the first $r_{1}$ 
embeddings represent the real primes and the remaining $r_{2}$ embeddings represent the complex primes. If $a \in K$, we denote by $a^{(i)}$ the $i^{\prime}$-th embedding of $a$. We may list the embeddings representing the infinite primes:

$$
\underbrace{a^{(1)}, \ldots, a^{\left(r_{1}\right)}}_{r_{1} \text { real }}, \underbrace{a^{\left(r_{1}+1\right)}, \ldots, a^{(n)}}_{r_{2} \text { complex }}
$$

where $n$ is the number of infinite primes of $K$. We let $O$ denote the ring of integers in $K$. Using the embeddings, we may identify $\operatorname{SL}(2, O)$ with its image $\Gamma \subset G$ under the injective homomorphism $\mathrm{SL}(2, K) \hookrightarrow G$ defined by

$$
\left(\begin{array}{ll}
a & b \\
c & d
\end{array}\right) \mapsto\left(\left(\begin{array}{ll}
a^{(1)} & b^{(1)} \\
c^{(1)} & d^{(1)}
\end{array}\right), \ldots,\left(\begin{array}{ll}
a^{(n)} & b^{(n)} \\
c^{(n)} & d^{(n)}
\end{array}\right)\right) .
$$

The image $\Gamma \subset G$ is called the Hilbert modular group associated to the field $K$. One can show that $\Gamma$ is a discrete, cofinite and irreducible subgroup of $G$. To explain the notion of irreducibility, we recall the notion of commensurable groups:

Definition 2.1. Two subgroups $\Gamma \subset G$ and $\Gamma^{\prime} \subset G$ are said to be strictly commensurable if the intersection $\Gamma \cap \Gamma^{\prime}$ has finite index in both $\Gamma$ and $\Gamma^{\prime}$. They are said to be commensurable if $\Gamma^{\prime}$ is strictly commensurable to $g \Gamma g^{-1}$ for some $g \in G$.

Commensurability and strict commensurability define equivalence relations on the set of subgroups of $G$. That $\Gamma$ is irreducible then means that it is not commensurable to a direct product $\Gamma_{1} \times \Gamma_{2}$, where $\Gamma_{1} \subset G_{1}$ and $\Gamma_{2} \subset G_{2}$ are discrete, $G_{1}$ and $G_{2}$ are nontrivial and $G=G_{1} \times G_{2}$. Conversely, in [7] it is shown that any discrete cofinite irreducible subgroup of $G$ is commensurable to a Hilbert modular group when $n>1$. There is a natural left action of $G$ on

$$
\mathbb{P}=\mathbb{P}^{1}(\mathbb{R})^{r_{1}} \times \mathbb{P}^{1}(\mathbb{C})^{r_{2}} .
$$

The cusps of $\Gamma$ are, by definition, the parabolic fixpoints in $\mathbb{P}$. It is easy to see that the set of cusps is exactly the image of the map $\mathbb{P}^{1}(K) \rightarrow \mathbb{P}$. The group $\Gamma$ acts on the set of cusps, and in fact there are only finitely many inequivalent cusps. Indeed, this number is equal to the ideal class number $h$.

Proposition 2.2. The number of inequivalent cusps for $\Gamma$ is equal to $h$.

Proof. As we have noted, the set of cusps is the image of the map $\mathbb{P}^{1}(K) \rightarrow \mathbb{P}$. We thus have a bijection

$$
\operatorname{SL}(2, O) \backslash \mathbb{P}^{1}(K) \approx \Gamma \backslash\{\text { cusps }\} .
$$

We are going to establish a bijection between $\operatorname{SL}(2, O) \backslash \mathbb{P}^{1}(K)$ and the ideal class group Cl: Each $(a: b) \in \mathbb{P}^{1}(K)$ is mapped to the class of the nonzero fractional ideal $a O+b O$. It is easy to see that this map factors and gives a surjective map

$$
\mathrm{SL}(2, O) \backslash \mathbb{P}^{1}(K) \rightarrow \mathrm{Cl} \text {. }
$$

To prove that this is injective, look at $(a: b) \in \mathbb{P}^{1}(K)$ and $\left(a^{\prime}: b^{\prime}\right) \in \mathbb{P}^{1}(K)$ with $a O+b O=a^{\prime} O+b^{\prime} O$ in Cl. We may assume that $a O+b O=a^{\prime} O+b^{\prime} O=\mathfrak{a}$. Then we can find $c, d \in \mathfrak{a}^{-1}$ and $c^{\prime}, d^{\prime} \in \mathfrak{a}^{-1}$ such that

$$
\sigma=\left(\begin{array}{ll}
a & c \\
b & d
\end{array}\right) \in \mathrm{SL}(2, K) \text { and } \sigma^{\prime}=\left(\begin{array}{ll}
a^{\prime} & c^{\prime} \\
b^{\prime} & d^{\prime}
\end{array}\right) \in \mathrm{SL}(2, K) .
$$

Now we have the following:

$$
\left(a^{\prime}, b^{\prime}\right)^{T}=\sigma^{\prime}(1,0)^{T}=\sigma^{\prime} \sigma^{-1}(a, b)^{T},
$$


and by multiplication, we see that $\sigma^{\prime} \sigma^{-1}$ has entries in $O$ and determinant 1 .

The proof gives us a canonical bijection $\Gamma \backslash\{$ cusps $\} \approx \mathrm{Cl}$ mapping the class of $(a: b) \in \mathbb{P}^{1}(K)$ to the ideal class of $a O+b O$. We will denote by $C_{\kappa}$ the ideal class associated to the cusp $\kappa$. The following proposition says that we may move an arbitrary cusp $\kappa$ to $\infty$ such that the isotropy groups $\Gamma_{\kappa}$ and $\Gamma_{\infty}$ are almost conjugate. In many cases, this allows us to consider only the cusp $\infty$.

Proposition 2.3. Let $\kappa \in \mathbb{P}$ be a cusp. Then there exists a $\sigma \in \operatorname{SL}(2, K) \subset G$ such that $\sigma \infty=\kappa$ and

$$
\sigma^{-1} \Gamma_{\kappa} \sigma=\left\{\left(\begin{array}{cc}
u & b \\
0 & u^{-1}
\end{array}\right): u \in O^{\times} \text {and } b \in \mathfrak{a}^{-2}\right\},
$$

for some integral ideal $\mathfrak{a} \in C_{\kappa}$. If $\bar{\sigma}$ is another such matrix and $\overline{\mathfrak{a}}$ is the corresponding ideal, then $\bar{\sigma}$ has the form

$$
\bar{\sigma}=\sigma\left(\begin{array}{cc}
a & * \\
0 & a^{-1}
\end{array}\right)
$$

where $a$ is a generator of the principal ideal $\mathfrak{a}^{-1} \overline{\mathfrak{a}}$.

Proof. Write $\kappa=(a: b)$ where $a, b \in O$ and put $\mathfrak{a}=a O+b O$. There exists $c, d \in \mathfrak{a}^{-1}$ such that

$$
\sigma=\left(\begin{array}{ll}
a & c \\
b & d
\end{array}\right) \in \mathrm{SL}(2, K)
$$

Clearly $\sigma \infty=\kappa$. To prove the statement about $\sigma^{-1} \Gamma_{\kappa} \sigma$, consider first a $\gamma \in \Gamma_{\kappa}$. Then

$$
\sigma^{-1} \gamma \sigma=\left(\begin{array}{cc}
u & b^{\prime} \\
0 & u^{-1}
\end{array}\right)
$$

for some $u \in K^{*}$ and $b^{\prime} \in K$. By looking at the trace, we conclude that $u \in O^{\times}$:

$$
u+u^{-1}=\operatorname{tr} \gamma \in O \Rightarrow u, u^{-1} \in O \Rightarrow u \in O^{\times} .
$$

By computing the upper right entry of $\sigma^{-1} \gamma \sigma$, it is straightforward to verify that $b^{\prime} \in \mathfrak{a}^{-2}$. Conversely, let $u \in O^{\times}$and $b^{\prime} \in \mathfrak{a}^{-2}$. Again, by doing the multiplications, it is straightforward to see that

$$
\sigma\left(\begin{array}{cc}
u & b^{\prime} \\
0 & u^{-1}
\end{array}\right) \sigma^{-1}
$$

fixes $\kappa$, has entries in $O$ and determinant 1. Thus

$$
\sigma^{-1} \Gamma_{\kappa} \sigma=\left\{\left(\begin{array}{cc}
u & b^{\prime} \\
0 & u^{-1}
\end{array}\right): u \in O^{\times} \text {and } b^{\prime} \in \mathfrak{a}^{-2}\right\} .
$$

It is also clear that the ideal class of $\mathfrak{a}$ is the class $C_{\kappa}$ associated to $\kappa$. This proves the existence. Now suppose $\bar{\sigma}$ is another matrix with the desired properties and let $\overline{\mathfrak{a}}$ be the corresponding ideal. Then

$$
\bar{\sigma}=\sigma\left(\begin{array}{cc}
a & b^{\prime} \\
0 & a^{-1}
\end{array}\right)
$$

for some $a \in K^{*}$ and $b^{\prime} \in K$. If we use this to express $\bar{\sigma}^{-1} \Gamma_{\kappa} \bar{\sigma}$ as a conjugate of $\sigma^{-1} \Gamma_{\kappa} \sigma$, we find that $\mathfrak{a}^{-2} \overline{\mathfrak{a}}^{2} \subset(a)^{2}$. Doing this the other way around, we find that $\mathfrak{a}^{-2} \overline{\mathfrak{a}}^{2} \supset(a)^{2}$. By unique factorization, we have $\mathfrak{a}^{-1} \overline{\mathfrak{a}}=(a)$. 
Any such matrix $\sigma$ as in the proposition is called a scaling matrix for the cusp $\kappa$. The integral ideal $\mathfrak{a}$ is uniquely determined by $\sigma$. Indeed, it is easy to see that $\mathfrak{a}$ is the ideal generated by the last row of $\sigma^{-1}$. We will denote the ideal $\mathfrak{a}^{-2}$ by $\mathfrak{b}$.

\section{EISENSTEIN SERIES}

In this section we will introduce the Eisenstein series on $\Gamma$ essentially as in [1. Let us begin by considering the cusp $\infty$. For $z \in X$, we should look at the series

$$
\sum_{\gamma \in \Gamma_{\infty} \backslash \Gamma} \prod_{i=1}^{n} y_{i}(\gamma z)^{s_{i}}
$$

for complex parameters $s_{i}$. However, for this to be well-defined, the $s_{i}$ should satisfy some condition. Let us explore this further. What we need is that each term should depend only on the class of $\gamma$ in $\Gamma_{\infty} \backslash \Gamma$. This happens if and only if

$$
\left|u^{(1)}\right|^{2 s_{1}} \cdots\left|u^{(n)}\right|^{2 s_{n}}=1,
$$

for all $u \in O^{\times}$. This is sufficient to check on some basis $u_{1}, \ldots, u_{\nu}$ for $O^{\times}$, where we have introduced the notation

$$
\nu=\operatorname{rk} O^{\times}=r_{1}+r_{2}-1=n-1 .
$$

We fix this basis for $O^{\times}$(and its ordering). Then the condition we need is that

$$
\left(\begin{array}{ccc}
\log \left|u_{1}^{(1)}\right| & \cdots & \log \left|u_{1}^{(n)}\right| \\
\cdots & \cdots & \cdots \\
\log \left|u_{\nu}^{(1)}\right| & \cdots & \log \left|u_{\nu}^{(n)}\right|
\end{array}\right)\left(\begin{array}{c}
s_{1} \\
\cdots \\
s_{n}
\end{array}\right) \in \pi i \mathbb{Z}^{\nu}
$$

Hence, consider fixed $s \in \mathbb{C}$ and $m \in \mathbb{Z}^{\nu}$. Then we want to solve the equation

$$
\left(\begin{array}{ccc}
\log \left|u_{1}^{(1)}\right| & \cdots & \log \left|u_{1}^{(n)}\right| \\
\cdots & \cdots & \cdots \\
\log \left|u_{\nu}^{(1)}\right| & \cdots & \log \left|u_{\nu}^{(n)}\right| \\
1 & \cdots & 1
\end{array}\right)\left(\begin{array}{c}
s_{1} \\
\cdots \\
s_{n}
\end{array}\right)=\left(\begin{array}{c}
\pi i m_{1} \\
\cdots \\
\pi i m_{\nu} \\
d s
\end{array}\right) .
$$

There is a unique solution. Indeed, the matrix in the equation is invertible as follows from

Lemma 3.1. Let $A=\left\{a_{i j}\right\}$ be an $n \times n$ matrix with real entries such that $\sum_{j=1}^{n} a_{i j}$ $=0$ for all $i \neq n$. Then $\operatorname{det}(A)$ does not change if the last row is replaced by any row with the same coordinate sum.

Proof. Let $B$ be the new matrix with the replaced row. We claim that $\operatorname{det}(B)=$ $\operatorname{det}(A)$; so look at the difference:

$$
\operatorname{det}(A)-\operatorname{det}(B)=\sum_{j=1}^{n}(-1)^{n+j}\left(a_{n j}-b_{n j}\right) \operatorname{det}\left(A_{n j}\right)=\operatorname{det}(C),
$$

where $C$ is a matrix where all rows have coodinate sum 0 . Thus the columns are not independent and hence $C$ is singular.

We can apply the lemma to the above matrix with the last $r_{2}$ columns multiplied by 2 . We replace the last row by $(0, \ldots, 0, d)$. Then it is easy to see that the 
determinant of the above matrix is $\pm 2^{-r_{2}} d R$ where $R$ is the regulator of $K$. Thus we may define real numbers $e_{i j}$ as follows:

$$
\left(\begin{array}{cccc}
e_{11} & \cdots & e_{1 \nu} & 1 / d \\
\cdots & \cdots & \cdots & \cdots \\
e_{n 1} & \cdots & e_{n \nu} & 2 / d
\end{array}\right)=\left(\begin{array}{ccc}
\log \left|u_{1}^{(1)}\right| & \ldots & \log \left|u_{1}^{(n)}\right| \\
\cdots & \cdots & \cdots \\
\log \left|u_{\nu}^{(1)}\right| & \ldots & \log \left|u_{\nu}^{(n)}\right| \\
1 & \cdots & 1
\end{array}\right)^{-1}
$$

The computation of $e_{i n}$ deserves an argument: Let $v=\left(e_{1 n}, \ldots, e_{n n}\right)^{T}$ be the last column. Clearly it is orthogonal to the vectors

$$
v_{i}=\left(\log \left|u_{i}^{(1)}\right|, \ldots, \log \left|u_{i}^{(n)}\right|\right)^{T},
$$

where $i=1, \ldots, \nu$. However, we have just seen that $v_{1}, \ldots, v_{\nu}$ are independent. Clearly, $\operatorname{Span}\left\{v_{1}, \ldots, v_{\nu}\right\}$ is contained in the complement of $(1, \ldots, 2)^{T}$. Looking at dimensions we see that $\operatorname{Span}\left\{v_{1}, \ldots, v_{\nu}\right\}$ in fact is the complement of $(1, \ldots, 2)^{T}$. Thus $v$ is a multiple of $(1, \ldots, 2)^{T}$, and since $\left\langle v,(1, \ldots, 1)^{T}\right\rangle=1$, we have $v=$ $1 / d(1, \ldots, 2)^{T}$. With this notation, the solution to the above equation is given by

$$
s_{i}=s_{i}(s, m)=s+\pi i\left\langle m, e_{i}\right\rangle=s+\pi i \sum_{j=1}^{\nu} m_{j} e_{i j}
$$

when $i \leq r$, where $e_{i}$ is the $i^{\prime}$-th row of the left $n \times \nu$ block of $\left\{e_{i j}\right\}$. When $i>r$, we must replace $s$ by $2 s$. Thus the Eisenstein series should have parameters $z \in X$, $s \in \mathbb{C}, m \in \mathbb{Z}^{\nu}$ and be given as

$$
\sum_{\gamma \in \Gamma_{\infty} \backslash \Gamma} \prod_{i=1}^{n} y_{i}(\gamma z)^{s_{i}}, \text { where } s_{i}=s_{i}(s, m) .
$$

When we consider an arbitrary cusp $\kappa$, the Eisenstein series should be

$$
\sum_{\gamma \in \Gamma_{\kappa} \backslash \Gamma} \prod_{i=1}^{n} y_{i}\left(\sigma^{-1} \gamma z\right)^{s_{i}}, \text { where } s_{i}=s_{i}(s, m),
$$

and $\sigma$ is a scaling matrix for $\kappa$. This still makes sense as a series. However, the series should not depend on the choice of $\sigma$. If $\bar{\sigma}$ is another scaling matrix for $\kappa$, we have seen that it has the form

$$
\bar{\sigma}=\sigma\left(\begin{array}{cc}
a & * \\
0 & a^{-1}
\end{array}\right)
$$

where $a$ is a generator of the principal ideal $\mathfrak{a}^{-1} \overline{\mathfrak{a}}$. Then (formally at least)

$$
\sum_{\gamma \in \Gamma_{\kappa} \backslash \Gamma} \prod_{i=1}^{n} y_{i}\left(\bar{\sigma}^{-1} \gamma z\right)^{s_{i}}=\frac{\chi_{m}(a)}{\mathrm{N}_{K / \mathbb{Q}}(a)^{2 s}} \sum_{\gamma \in \Gamma_{\kappa} \backslash \Gamma} \prod_{i=1}^{n} y_{i}\left(\sigma^{-1} \gamma z\right)^{s_{i}},
$$

where we have introduced the character $\chi_{m}:\left(\mathbb{R}^{*}\right)^{r_{1}} \times\left(\mathbb{C}^{*}\right)^{r_{2}} \rightarrow \mathbb{C}^{1}$ by

$$
\chi_{m}\left(x_{1}, \ldots, x_{n}\right)=\prod_{i=1}^{n}\left|x_{i}\right|^{-2 \pi i\left\langle m, e_{i}\right\rangle} .
$$

This is trivial on $O^{\times}$, and hence we may consider $\chi_{m}$ as a character on the group of principal ideals $P$. We may extend this character (in $h$ ways) to a character 
$\chi: I \rightarrow S^{1}$. This is a Grössencharakter modulo $\mathfrak{m}=O$. No matter what extension we choose, we have the equality

$$
\frac{\mathrm{N}_{K / \mathbb{Q}}(\overline{\mathfrak{a}})^{2 s}}{\chi(\overline{\mathfrak{a}})} \sum_{\gamma \in \Gamma_{\kappa} \backslash \Gamma} \prod_{i=1}^{n} y_{i}\left(\bar{\sigma}^{-1} \gamma z\right)^{s_{i}}=\frac{\mathrm{N}_{K / \mathbb{Q}}(\mathfrak{a})^{2 s}}{\chi(\mathfrak{a})} \sum_{\gamma \in \Gamma_{\kappa} \backslash \Gamma} \prod_{i=1}^{n} y_{i}\left(\sigma^{-1} \gamma z\right)^{s_{i}} .
$$

Thus we are led to the following definition of the Eisenstein series.

Definition 3.2. Let $\kappa \in \mathbb{P}$ be a cusp. For $z \in X, s \in \mathbb{C}, m \in \mathbb{Z}^{\nu}$ and a Grössencharakter $\chi$ modulo $\mathfrak{m}=O$ extending $\chi_{m}$, the Eisenstein series $E_{\kappa}(z, s$, $m, \chi)$ is defined as follows. Choose a scaling matrix $\sigma$ for $\kappa$ with ideal $\mathfrak{a}$, and put $s_{i}=s_{i}(s, m)$. Then

$$
E_{\kappa}(z, s, m, \chi)=\frac{\mathrm{N}_{K / \mathbb{Q}}(\mathfrak{a})^{2 s}}{\chi(\mathfrak{a})} \sum_{\gamma \in \Gamma_{\kappa} \backslash \Gamma} \prod_{i=1}^{n} y_{i}\left(\sigma^{-1} \gamma z\right)^{s_{i}}
$$

where $\gamma$ runs through a complete set of inequivalent coset representatives of $\Gamma_{\kappa} \backslash \Gamma$.

This definition is slightly different from the one in [1]. Note that $E_{\kappa}(z, s, m, \chi)$ depend on the ordered basis for $O^{\times}$that we have chosen. If we choose another ordered basis $u_{1}^{\prime}, \ldots, u_{\nu}^{\prime}$ and define $\chi_{m}^{\prime}$ and the Eisenstein series $E_{\kappa}^{\prime}(z, s, m, \chi)$ as above, then it is easy to see that there exists $A \in \mathrm{GL}_{\nu}(\mathbb{Z})$ such that $\chi_{m}^{\prime}=\chi_{A m}$ and $E_{\kappa}^{\prime}(z, s, m, \chi)=E_{\kappa}(z, s, A m, \chi)$ for any $\chi$ extending $\chi_{m}^{\prime}$. It is also straightforward to verify that if $\kappa$ and $\kappa^{\prime}$ are equivalent cusps, then $E_{\kappa^{\prime}}(z, s, m, \chi)=$ $E_{\kappa}(z, s, m, \chi)$. By the same arguments as in [1], one can show that the Eisenstein series $E_{\kappa}(z, s, m, \chi)$ converges absolutely for $\operatorname{Re}(s)>>0$ uniformly on compact subsets. The $E_{\kappa}(z, s, m, \chi)$ have meromorphic continuation to all $s \in \mathbb{C}$ and they satisfy a functional equation as follows: Choose a complete set of inequivalent cusps, put an order on them and arrange them in a column vector

$$
\mathfrak{E}(z, s, m, \chi)=\left\{E_{\kappa}(z, s, m, \chi)\right\} .
$$

Then one can show that there exists a matrix $\Phi(s, m, \chi)$ with meromorphic entries such that

$$
\mathfrak{E}(z, s, m, \chi)=\Phi(s, m, \chi) \mathfrak{E}(z, 1-s,-m, \bar{\chi}) .
$$

In fact, we shall derive this from the corresponding properties of partial Hecke $L$-series. The matrix $\Phi(s, m, \chi)$ is called the scattering matrix (or Eisenstein matrix), and it plays a fundamental role in the spectral theory of automorphic forms. From the functional equation, it follows immediately that $\Phi(s, m, \chi)$ itself satisfies a functional equation:

$$
\Phi(s, m, \chi) \Phi(1-s,-m, \bar{\chi})=I_{h} .
$$

Later we identify the entries of $\Phi(s, m, \chi)$ in the constant terms of the Fourier expansions of the Eisenstein series.

\section{Normalization of the Eisenstein SERIES}

As a function of $z \in X$, the Eisenstein series $E_{\kappa}(z, s, m, \chi)$ is a $\Gamma$-automorphic smooth eigenfunction of the Laplacians in each coordinate. For $i \leq r_{1}$, these are given by

$$
\Delta_{i}=y_{i}^{2}\left(\frac{\partial^{2}}{\partial x_{i}^{2}}+\frac{\partial^{2}}{\partial y_{i}^{2}}\right)
$$


while for $i>r_{1}$, they are given by

$$
\Delta_{i}=y_{i}^{2}\left(\frac{\partial^{2}}{\partial x_{i} \partial \bar{x}_{i}}+\frac{\partial^{2}}{\partial y_{i}^{2}}\right)-y_{i} \frac{\partial}{\partial y_{i}} .
$$

They are clearly invariant. A general theorem says that a diffeomorphism of a Riemannian manifold is an isometry if and only if it leaves the Laplace operator invariant. They are the fundamental operators in the terminology of [6]. We want to study the asymptotics of $E_{\kappa}(z, s, m, \chi)$ as $z \rightarrow \kappa^{\prime}$ within a fundamental domain. We do this by choosing a scaling matrix $\sigma^{\prime}$ for $\kappa^{\prime}$, and look at $E_{\kappa}\left(\sigma^{\prime} z, s, m, \chi\right)$ as $z \rightarrow \infty$. Only the dependence of $y=\left(y_{1}, \ldots, y_{n}\right) \in \mathbb{R}_{+}^{n}$ is important, since $x=\left(x_{1}, \ldots, x_{n}\right) \in \mathbb{R}^{r_{1}} \times \mathbb{C}^{r_{2}}$ is bounded within a fundamental domain. Thus we fix a $y \in \mathbb{R}_{+}^{n}$, and look at $E_{\kappa}\left(\sigma^{\prime} z, s, m, \chi\right)$ as a function of $x \in \mathbb{R}^{r_{1}} \times \mathbb{C}^{r_{2}}$. Since Eisenstein series are automorphic, we find that it is $\mathfrak{b}^{\prime}$-periodic where $\mathfrak{b}^{\prime}$ is the ideal of $\sigma^{\prime}$ thought of as a complete lattice in $\mathbb{R}^{r_{1}} \times \mathbb{C}^{r_{2}}$. Hence it has a Fourier expansion

$$
E_{\kappa}\left(\sigma^{\prime} z, s, m, \chi\right)=\sum_{l \in \mathfrak{b}^{\prime *}} a_{l}^{\kappa, \kappa^{\prime}}(y, s, m, \chi) e^{2 \pi i \operatorname{Tr}_{K / \mathbb{Q}}(l x)},
$$

where $\mathfrak{b}^{\prime *}=\left\{l \in K: \operatorname{Tr}_{K / \mathbb{Q}}\left(l \mathfrak{b}^{\prime}\right) \subset \mathbb{Z}\right\}$ is the dual module and

$$
\operatorname{Tr}_{K / \mathbb{Q}}(x)=\sum_{i \leq r_{1}} x_{i}+\sum_{i>r_{1}} 2 \operatorname{Re}\left(x_{i}\right)
$$

for $x=\left(x_{1}, \ldots, x_{n}\right) \in \mathbb{R}^{r_{1}} \times \mathbb{C}^{r_{2}}$. The Fourier coefficients are given by

$$
a_{l}^{\kappa, \kappa^{\prime}}(y, s, m, \chi)=\frac{2^{r_{2}}}{\mathrm{~N}_{K / \mathbb{Q}}\left(\mathfrak{b}^{\prime}\right) \sqrt{|D|}} \int_{F_{\mathfrak{b}^{\prime}}} E_{\kappa}\left(\sigma^{\prime} z, s, m, \chi\right) e^{-2 \pi i \operatorname{Tr}_{K / \mathbb{Q}}(l x)} d x,
$$

where $F_{\mathfrak{b}^{\prime}}$ is a fundamental mesh for the lattice, $D$ is the discriminant of $K$ and $d x$ is the usual Lebesgue measure on $\mathbb{R}^{r_{1}} \times \mathbb{C}^{r_{2}}$. We use this notation, keeping in mind that $a_{l}^{\kappa, \kappa^{\prime}}(y, s, m, \chi)$ depends on the choice of a scaling matrix

$$
\sigma^{\prime}=\left(\begin{array}{ll}
a^{\prime} & c^{\prime} \\
b^{\prime} & d^{\prime}
\end{array}\right) \in \mathrm{SL}(2, K) \subset G
$$

for $\kappa^{\prime}$. The main result of this paper is the computation of the Fourier coefficients above.

Lemma 4.1. Let $\sigma$ be a scaling matrix for $\kappa$ with ideal $\mathfrak{a}$. Then

$$
E_{\kappa}\left(\sigma^{\prime} z, s, m, \chi\right)=\frac{\mathrm{N}_{K / \mathbb{Q}}(\mathfrak{a})^{2 s}}{\chi(\mathfrak{a})} \sum_{\substack{(C, D)^{\times} \\\left(C d^{\prime}-D b^{\prime}, C c^{\prime}-D a^{\prime}\right)=\mathfrak{a}}} \prod_{i=1}^{n} \frac{y_{i}^{s_{i}}}{\left|C^{(i)} z_{i}+D^{(i)}\right|^{2 s_{i}}}
$$

where $(C, D)^{\times}$denotes $O^{\times}$-equivalence.

Proof. Note that the group $\sigma^{-1} \Gamma_{\kappa} \sigma$ acts on the set $\sigma^{-1} \Gamma \sigma^{\prime}$, and we can write

$$
E_{\kappa}\left(\sigma^{\prime} z, s, m, \chi\right)=\frac{\mathrm{N}_{K / \mathbb{Q}}(\mathfrak{a})^{2 s}}{\chi(\mathfrak{a})} \sum_{\gamma \in \sigma^{-1} \Gamma_{\kappa} \sigma \backslash \sigma^{-1} \Gamma \sigma^{\prime}} \prod_{i=1}^{n} y_{i}(\gamma z)^{s_{i}} .
$$

The map that associates the last row to a $\gamma \in \sigma^{-1} \Gamma \sigma^{\prime}$ factors and gives a bijection

$$
\sigma^{-1} \Gamma_{\kappa} \sigma \backslash \sigma^{-1} \Gamma \sigma^{\prime} \approx\left\{\begin{array}{c}
\text { Pairs }(C, D) \text { of } C, D \in K \\
\text { s.t. }(A, B, C, D) \in \sigma^{-1} \Gamma \sigma^{\prime} \\
\text { for some } A, B \in K
\end{array}\right\} / O^{\times} .
$$


Thus, we may write

$$
E_{\kappa}\left(\sigma^{\prime} z, s, m, \chi\right)=\frac{\mathrm{N}_{K / \mathbb{Q}}(\mathfrak{a})^{2 s}}{\chi(\mathfrak{a})} \sum_{\substack{(C, D)^{\times} \\(A, B, C, D) \in \sigma^{-1} \Gamma \sigma^{\prime}}} \prod_{i=1}^{n} \frac{y_{i}^{s_{i}}}{\left|C^{(i)} z_{i}+D^{(i)}\right|^{2 s_{i}}} .
$$

Now, from the section on the cusps we know that

$$
\exists A, B:\left(\begin{array}{cc}
A & B \\
C & D
\end{array}\right) \in \sigma^{-1} \Gamma \sigma^{\prime} \Leftrightarrow\left(C d^{\prime}-D b^{\prime}, C c^{\prime}-D a^{\prime}\right)=\mathfrak{a},
$$

since $\mathfrak{a}$ is the ideal generated by the last row of $\sigma^{-1}$.

Let us briefly recall the definition of the partial $L$-series $L(s, \chi, C)$, where $\chi$ : $I \rightarrow S^{1}$ is a Grössencharakter modulo $\mathfrak{m}=O$ extending $\chi_{m}$, and $C \in \mathrm{Cl}$ is an ideal class:

$$
L(s, \chi, C)=\sum_{\substack{\mathfrak{a} \in C \\ \text { integral }}} \frac{\chi(\mathfrak{a})}{\mathrm{N}_{K / \mathbb{Q}}(\mathfrak{a})^{s}}
$$

The series is absolutely convergent for $\operatorname{Re}(s)>1$, and uniformly in $\operatorname{Re}(s)>1+\epsilon$ for every $\epsilon>0$. Thus $L(s, \chi, C)$ defines an analytic function for $\operatorname{Re}(s)>1$. We define the completed partial $L$-series by

$$
\Lambda(2 s, \chi, C)=2^{r_{2}+\left(s_{1}+\cdots+s_{r_{1}}\right)}(2 \pi)^{-d s}|D|^{s}\left\{\prod_{i=1}^{n} \Gamma\left(s_{i}\right)\right\} L(2 s, \chi, C),
$$

where $s_{i}=s_{i}(s, m)$. It is shown in [5] that $\Lambda(s, \chi, C)$ has a meromorphic continuation to the whole complex plane, and a functional equation

$$
\Lambda(s, \chi, C)=\chi(\mathfrak{d}) \Lambda\left(1-s, \bar{\chi}, C^{\prime}\right),
$$

where $C C^{\prime}=[\mathfrak{d}]$, is the class of the different $\mathfrak{d}$. Recall that, by definition, $\mathfrak{d}^{-1}=O^{*}$. We fix a complete set of inequivalent cusps, put an order on it and arrange the partial $L$-series in an $h \times h$ matrix as follows:

$$
\mathbb{L}(s, \chi)=\left\{L\left(s, \chi, C_{\kappa}^{-1} C_{\kappa^{\prime}}\right)\right\} .
$$

We also fix scaling matrices for each of the cusps, and put

$$
\mathbb{E}(z, s, m, \chi)=\left\{E_{\kappa}\left(\sigma^{\prime} z, s, m, \chi\right)\right\} .
$$

We always assume that $\infty$ is in our set of inequivalent cusps, and that the fixed scaling matrix there is 1 . Then we have the following analogue of Proposition 2.5 in 2]:

Lemma 4.2. Let $\sigma$ be a scaling matrix for $\kappa$ with ideal $\mathfrak{a}$. Then

$$
(\mathbb{L}(2 s, \chi) \mathbb{E}(z, s, m, \chi))_{\kappa \kappa^{\prime}}=\frac{\mathrm{N}_{K / \mathbb{Q}}(\mathfrak{a})^{2 s}}{\chi(\mathfrak{a})} \sum_{\substack{(C, D)^{\times} \\ 0 \neq\left(C d^{\prime}-D b^{\prime}, C c^{\prime}-D a^{\prime}\right) \subset \mathfrak{a}}} \prod_{i=1}^{n} \frac{y_{i}^{s_{i}}}{\left|C^{(i)} z_{i}+D^{(i)}\right|^{2 s_{i}}}
$$

where $(C, D)^{\times}$denotes $O^{\times}$-equivalence.

Proof. The right-hand side is equal to

$$
\frac{\mathrm{N}_{K / \mathbb{Q}}(\mathfrak{a})^{2 s}}{\chi(\mathfrak{a})} \sum_{\mathfrak{a} \mid \mathfrak{q}} \sum_{\substack{(C, D)^{\times} \\\left(C d^{\prime}-D b^{\prime}, C c^{\prime}-D a^{\prime}\right)=\mathfrak{q}}} \prod_{i=1}^{n} \frac{y_{i}^{s_{i}}}{\left|C^{(i)} z_{i}+D^{(i)}\right|^{2 s_{i}}} .
$$


We divide the first sum into ideal classes and obtain

$$
\frac{\mathrm{N}_{K / \mathbb{Q}}(\mathfrak{a})^{2 s}}{\chi(\mathfrak{a})} \sum_{\kappa^{\prime \prime}} \sum_{\substack{\mathfrak{a} \mid \mathfrak{q} \\ \mathfrak{q} \in C_{\kappa^{\prime \prime}}}} \sum_{\substack{(C, D)^{\times} \\\left(C d^{\prime}-D b^{\prime}, C c^{\prime}-D a^{\prime}\right)=\mathfrak{q}}} \prod_{i=1}^{n} \frac{y_{i}^{s_{i}}}{\left|C^{(i)} z_{i}+D^{(i)}\right|^{2 s_{i}}},
$$

where $\kappa^{\prime \prime}$ runs through the complete set of inequivalent cusps. Writing $\mathfrak{q}$ as $\mathfrak{q}=$ $\mathfrak{a}^{\prime \prime}(\theta)$, where $\theta \in \mathfrak{a}^{\prime \prime-1} \mathfrak{a}$, we find that this equals

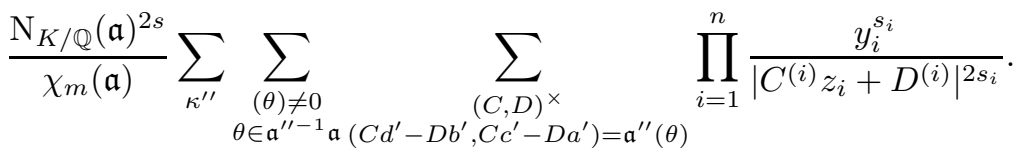

Changing variables $C \rightarrow C / \theta$ and $D \rightarrow D / \theta$, we get

$$
\frac{\mathrm{N}_{K / \mathbb{Q}}(\mathfrak{a})^{2 s}}{\chi(\mathfrak{a})} \sum_{\kappa^{\prime \prime}} \sum_{\substack{(\theta) \neq \neq=0 \\ \theta \in \mathfrak{a}^{\prime \prime-1} \mathfrak{a}}} \frac{\chi(\theta)}{\mathrm{N}_{K / \mathbb{Q}}(\theta)^{2 s}} \sum_{\substack{(C, D)^{\times} \\\left(C d^{\prime}-D b^{\prime}, C c^{\prime}-D a^{\prime}\right)=\mathfrak{a}^{\prime \prime}}} \prod_{i=1}^{n} \frac{y_{i}^{s_{i}}}{\left|C^{(i)} z_{i}+D^{(i)}\right|^{2 s_{i}}} .
$$

Using the lemma above, and the fact that $\mathfrak{a}^{-1} \mathfrak{a}^{\prime \prime}(\theta)$ runs through all integral ideals in the class $C_{\kappa}^{-1} C_{\kappa^{\prime \prime}}$ as $\theta \in \mathfrak{a}^{\prime \prime-1} \mathfrak{a}$, we see that this is equal to the left-hand side.

As we see from Lemma 4.1, the Eisenstein series can be written as

$$
E_{\kappa}(z, s, m, \chi)=\frac{\mathrm{N}_{K / \mathbb{Q}}(\mathfrak{a})^{2 s}}{\chi(\mathfrak{a})} \sum_{\substack{(C, D)^{\times} \\(C, D)=\mathfrak{a}}} \prod_{i=1}^{n} \frac{y_{i}^{s_{i}}}{\left|C^{(i)} z_{i}+D^{(i)}\right|^{2 s_{i}}} .
$$

We define the normalized Eisenstein series to be

$$
E_{\kappa}^{*}(z, s, m, \chi)=\frac{\mathrm{N}_{K / \mathbb{Q}}(\mathfrak{a})^{2 s}}{\chi(\mathfrak{a})} \sum_{\substack{(C, D)^{\times} \\ 0 \neq(C, D) \subset \mathfrak{a}}} \prod_{i=1}^{n} \frac{y_{i}^{s_{i}}}{\left|C^{(i)} z_{i}+D^{(i)}\right|^{2 s_{i}}} .
$$

From Lemma 4.2, it follows that

$$
\mathbb{E}^{*}(z, s, m, \chi)=\mathbb{L}(2 s, \chi) \mathbb{E}(z, s, m, \chi),
$$

where $\mathbb{E}^{*}(z, s, m, \chi)$ is the matrix with entries $E_{\kappa}^{*}\left(\sigma^{\prime} z, s, m, \chi\right)$. We also define the completed Eisenstein series to be

$$
\hat{E}_{\kappa}(z, s, m, \chi)=2^{r_{2}+\left(s_{1}+\cdots+s_{r_{1}}\right)}(2 \pi)^{-d s}|D|^{s}\left\{\prod_{i=1}^{n} \Gamma\left(s_{i}\right)\right\} E_{\kappa}^{*}(z, s, m, \chi) .
$$

Then we have the identity

$$
\hat{\mathbb{E}}(z, s, m, \chi)=\hat{\Lambda}(2 s, \chi) \mathbb{E}(z, s, m, \chi),
$$

where $\hat{\mathbb{E}}(z, s, m, \chi)$ is the matrix with entries $\hat{E}_{\kappa}\left(\sigma^{\prime} z, s, m, \chi\right)$, and

$$
\hat{\Lambda}(s, \chi)=\left\{\Lambda\left(s, \chi, C_{\kappa}^{-1} C_{\kappa^{\prime}}\right)\right\} .
$$

In particular, $\hat{E}_{\kappa}\left(\sigma^{\prime} z, s, m, \chi\right)$ is $\mathfrak{b}^{\prime}$-periodic. We compute the Fourier expansion of it, and from this we get the Fourier expansion of $E_{\kappa}\left(\sigma^{\prime} z, s, m, \chi\right)$. 


\section{The COnstant term of the Eisenstein SERIES}

We consider the normalized Eisenstein series at the cusp $\kappa^{\prime}$. We have

$$
E_{\kappa}^{*}\left(\sigma^{\prime} z, s, m, \chi\right)=\frac{\mathrm{N}_{K / \mathbb{Q}}(\mathfrak{a})^{2 s}}{\chi(\mathfrak{a})} \sum_{\substack{(C, D)^{\times} \\ 0 \neq\left(C d^{\prime}-D b^{\prime}, C c^{\prime}-D a^{\prime}\right) \subset \mathfrak{a}}} \prod_{i=1}^{n} \frac{y_{i}^{s_{i}}}{\left|C^{(i)} z_{i}+D^{(i)}\right|^{2 s_{i}}} .
$$

Since $\mathbb{E}^{*}(z, s, m, \chi)=\mathbb{L}(2 s, \chi) \mathbb{E}(z, s, m, \chi)$, this is $\mathfrak{b}^{\prime}$-periodic. Hence,

$$
E_{\kappa}^{*}\left(\sigma^{\prime} z, s, m, \chi\right)=\sum_{l \in \mathfrak{b}^{\prime *}} a_{l}^{\kappa, \kappa^{\prime}}(y, s, m, \chi)^{*} e^{2 \pi i \operatorname{Tr}_{K / \mathbb{Q}}(l x)},
$$

where the Fourier coefficients are given by

$$
a_{l}^{\kappa, \kappa^{\prime}}(y, s, m, \chi)^{*}=\frac{2^{r_{2}}}{\mathrm{~N}_{K / \mathbb{Q}}\left(\mathfrak{b}^{\prime}\right) \sqrt{|D|}} \int_{F_{\mathfrak{b}^{\prime}}} E_{\kappa}^{*}\left(\sigma^{\prime} z, s, m, \chi\right) e^{-2 \pi i \operatorname{Tr}_{K / \mathbb{Q}}(l x)} d x .
$$

To compute this, we divide the series $E_{\kappa}^{*}\left(\sigma^{\prime} z, s, m, \chi\right)$ into the terms with $C=0$ and those with $C \neq 0$. The terms with $C=0$ give a contribution

$$
\frac{\mathrm{N}_{K / \mathbb{Q}}\left(\mathfrak{a}^{\prime}\right)^{2 s}}{\chi\left(\mathfrak{a}^{\prime}\right)} L\left(2 s, \chi, C_{\kappa}^{-1} C_{\kappa^{\prime}}\right) y_{1}^{s_{1}} \cdots y_{n}^{s_{n}}
$$

to $E_{\kappa}^{*}\left(\sigma^{\prime} z, s, m, \chi\right)$. Now consider the terms with $C \neq 0$. We have a bijection

$$
\left\{\begin{array}{c}
\text { Pairs }(C, D) \text { of } C, D \in K \text { s.t. } \\
\left(C d^{\prime}-D b^{\prime}, C c^{\prime}-D a^{\prime}\right) \subset \mathfrak{a} \\
\text { and } C \neq 0
\end{array}\right\} / O^{\times} \approx\left\{\begin{array}{c}
\text { Pairs }(\mathfrak{q}, \eta) \text { with } \\
0 \neq \mathfrak{q} \subset \mathfrak{a} \mathfrak{a}^{\prime} \text { principal } \\
\text { and } \eta \in \mathfrak{a} \mathfrak{a}^{\prime-1} \mathfrak{q}^{-1}
\end{array}\right\},
$$

defined by mapping $(C, D)^{\times}$to $((C), D / C)$. Thus we change variables, and the contribution from the terms with $C \neq 0$ can be written

$$
\frac{\mathrm{N}_{K / \mathbb{Q}}(\mathfrak{a})^{2 s}}{\chi(\mathfrak{a})} \sum_{\substack{(C) \neq 0 \neq \\ C \in \mathfrak{a} \mathfrak{a}^{\prime}}} \frac{\chi(C)}{\mathrm{N}_{K / \mathbb{Q}}(C)^{2 s}} \sum_{\eta \in \mathfrak{a} \mathfrak{a}^{\prime-1}(C)^{-1}} \prod_{i=1}^{n} \frac{y_{i}^{s_{i}}}{\left|z_{i}+\eta^{(i)}\right|^{2 s_{i}}} .
$$

Since $C \in \mathfrak{a a}^{\prime}$, we have $\mathfrak{b}^{\prime} \subset \mathfrak{a a}^{\prime-1}(C)^{-1}$. We fix some set of representatives $\eta \in \mathfrak{a a}^{\prime-1}(C)^{-1} / \mathfrak{b}^{\prime}$, and we write the above as

$$
\frac{\mathrm{N}_{K / \mathbb{Q}}(\mathfrak{a})^{2 s}}{\chi(\mathfrak{a})} \sum_{\substack{(C) \neq 0 \\ C \in \mathfrak{a} \mathfrak{a}^{\prime}}} \frac{\chi(C)}{\mathrm{N}_{K / \mathbb{Q}}(C)^{2 s}} \sum_{\eta \in \mathfrak{a a}^{\prime}-1(C)^{-1} / \mathfrak{b}^{\prime}} \sum_{\alpha \in \mathfrak{b}^{\prime}} \prod_{i=1}^{n} \frac{y_{i}^{s_{i}}}{\left|z_{i}+\eta^{(i)}+\alpha^{(i)}\right|^{2 s_{i}}} .
$$

Now we can compute the constant term $a_{0}^{\kappa, \kappa^{\prime}}(y, s, m, \chi)^{*}$. The contribution from the terms with $C=0$ does not depend on $x$, so that when we integrate it to get the Fourier coefficient we obtain

$$
\frac{\mathrm{N}_{K / \mathbb{Q}}\left(\mathfrak{a}^{\prime}\right)^{2 s}}{\chi\left(\mathfrak{a}^{\prime}\right)} L\left(2 s, \chi, C_{\kappa}^{-1} C_{\kappa^{\prime}}\right) y_{1}^{s_{1}} \cdots y_{n}^{s_{n}}
$$

However, when we integrate the contribution from the terms with $C \neq 0$, we get

$$
\frac{\chi\left(\mathfrak{a}^{\prime}\right)}{\mathrm{N}_{K / \mathbb{Q}}\left(\mathfrak{a}^{\prime}\right)^{2 s-2}} \cdot \frac{2^{r_{2}}}{\sqrt{|D|}} L\left(2 s-1, \chi, C_{\kappa}^{-1} C_{\kappa^{\prime}}^{-1}\right) \prod_{i=1}^{n} \int \frac{y_{i}^{s_{i}} d x_{i}}{\left(\left|x_{i}\right|^{2}+y_{i}^{2}\right)^{s_{i}}} .
$$

To end the computation we need to compute the integrals. For $i \leq r_{1}$, we have

$$
\int_{-\infty}^{+\infty} \frac{y_{i}^{s_{i}} d x_{i}}{\left(x_{i}^{2}+y_{i}^{2}\right)^{s_{i}}}=\pi^{1 / 2} \frac{\Gamma\left(s_{i}-1 / 2\right)}{\Gamma\left(s_{i}\right)} y_{i}^{1-s_{i}}
$$


See for example 4]. The case $i>r_{1}$ is much easier:

$$
\int_{0}^{2 \pi} d \theta_{i} \int_{0}^{\infty} \frac{y_{i}^{s_{i}} r_{i} d r_{i}}{\left(r_{i}^{2}+y_{i}^{2}\right)^{s_{i}}}=\frac{\pi}{s_{i}-1} y_{i}^{2-s_{i}}
$$

Thus the contribution to ${ }^{*} a_{0}^{\kappa, \kappa^{\prime}}(y, s, m, \chi)$ from the terms with $C \neq 0$ is

$$
\begin{aligned}
\frac{\chi\left(\mathfrak{a}^{\prime}\right)}{\mathrm{N}_{K / \mathbb{Q}}\left(\mathfrak{a}^{\prime}\right)^{2 s-2}} \cdot \frac{2^{r_{2}} \pi^{\frac{d}{2}}}{\sqrt{|D|}}\left\{\prod_{i \leq r_{1}} \frac{\Gamma\left(s_{i}-\frac{1}{2}\right)}{\Gamma\left(s_{i}\right)} \prod_{i>r_{1}} \frac{1}{s_{i}-1}\right\} \\
\times L\left(2 s-1, \chi, C_{\kappa}^{-1} C_{\kappa^{\prime}}^{-1}\right) y_{1}^{1-s_{1}} \cdots y_{n}^{2-s_{n}} .
\end{aligned}
$$

Thus we have computed $* a_{0}^{\kappa, \kappa^{\prime}}(y, s, m, \chi)$. We rewrite this in terms of the completed partial $L$-series. It is easy to see that for any ideal class $C$,

$$
\frac{\Lambda(2 s-1, \chi, C)}{\Lambda(2 s, \chi, C)}=\frac{2^{r_{2}} \pi^{d / 2}}{\sqrt{|D|}}\left\{\prod_{i \leq r_{1}} \frac{\Gamma\left(s_{i}-1 / 2\right)}{\Gamma\left(s_{i}\right)} \prod_{i>r_{1}} \frac{1}{s_{i}-1}\right\} \frac{L(2 s-1, \chi, C)}{L(2 s, \chi, C)} .
$$

If we let $\hat{a}_{0}^{\kappa, \kappa^{\prime}}(y, s, m, \chi)$ denote the constant term of $\hat{E}_{\kappa}\left(\sigma^{\prime} z, s, m, \chi\right)$, then the above shows that it is a sum of a contribution

$$
\frac{\mathrm{N}_{K / \mathbb{Q}}\left(\mathfrak{a}^{\prime}\right)^{2 s}}{\chi\left(\mathfrak{a}^{\prime}\right)} \Lambda\left(2 s, \chi, C_{\kappa}^{-1} C_{\kappa^{\prime}}\right) y_{1}^{s_{1}} \cdots y_{n}^{s_{n}}
$$

and a contribution from the terms with $C \neq 0$ given by

$$
\frac{\chi\left(\mathfrak{a}^{\prime}\right)}{\mathrm{N}_{K / \mathbb{Q}}\left(\mathfrak{a}^{\prime}\right)^{2 s-2}} \cdot \Lambda\left(2 s-1, \chi, C_{\kappa}^{-1} C_{\kappa^{\prime}}^{-1}\right) y_{1}^{1-s_{1}} \cdots y_{n}^{2-s_{n}}
$$

To get $a_{0}^{\kappa, \kappa^{\prime}}(y, s, m, \chi)$, we interpret the above as a matrix equation. We arrange all the constant terms in a matrix

$$
a_{0}(y, s, m, \chi)=\left\{a_{0}^{\kappa, \kappa^{\prime}}(y, s, m, \chi)\right\},
$$

and similarly we define $\hat{a}_{0}(y, s, m, \chi)$. We let $\mathbb{P}$ denote the permutation matrix corresponding to inversion on $\mathrm{Cl}$,

$$
\mathbb{P}=\left\{\delta_{\kappa \iota\left(\kappa^{\prime}\right)}\right\},
$$

where $\iota$ is the permutation of the $\{\kappa\}$ corresponding to inversion on the class group:

$$
C_{\iota(\kappa)}=C_{\kappa}^{-1} \text {. }
$$

Then what we have proved above is the following

Lemma 5.1. Let $\kappa^{\prime}$ be a fixed cusp. The constant terms are given by

$a_{0}^{\kappa^{\prime}}(y, s, m, \chi)=e^{\kappa^{\prime}} \frac{\mathrm{N}_{K / \mathbb{Q}}\left(\mathfrak{a}^{\prime}\right)^{s}}{\chi\left(\mathfrak{a}^{\prime}\right)} y_{1}^{s_{1}} \cdots y_{n}^{s_{n}}+\frac{\chi\left(\mathfrak{a}^{\prime}\right)}{\mathrm{N}_{K / \mathbb{Q}}\left(\mathfrak{a}^{\prime}\right)^{2 s-2}} \Phi^{\kappa^{\prime}}(s, m, \chi) y_{1}^{1-s_{1}} \cdots y_{n}^{2-s_{n}}$ where $\Phi^{\kappa^{\prime}}(s, m, \chi)$ is the $\kappa^{\prime}$-th column of

$$
\Phi(s, m, \chi)=\hat{\Lambda}(2 s, \chi)^{-1} \hat{\Lambda}(2 s-1, \chi) \mathbb{P},
$$

with notation as above.

Later, when we have computed the remaining terms of the Eisenstein series, we find that the matrix $\Phi(s, m, \chi)$, in fact, is the scattering matrix. 


\section{The Remaining terms of the Eisenstein Series}

We now compute the Fourier coefficients $a_{l}^{\kappa, \kappa^{\prime}}(y, s, m, \chi)^{*}$ for nonzero $l \in \mathfrak{b}^{\prime *}$. As before we divide the series $E_{\kappa}^{*}\left(\sigma^{\prime} z, s, m, \chi\right)$ into the terms with $C=0$ and those with $C \neq 0$. The terms with $C=0$ give no contribution. The other terms give

$$
\frac{\chi\left(\mathfrak{a}^{\prime}\right)}{\mathrm{N}_{K / \mathbb{Q}}\left(\mathfrak{a}^{\prime}\right)^{2 s-2}} \cdot \frac{2^{r_{2}}}{\sqrt{|D|}} \sigma_{1-2 s}^{\kappa, \kappa^{\prime}}(\chi, l) \int \prod_{i=1}^{n} \frac{y_{i}^{s_{i}} e^{-2 \pi i \operatorname{Tr}_{K / \mathbb{Q}}(l x)}}{\left(\left|x_{i}\right|^{2}+y_{i}^{2}\right)^{s_{i}}} d x
$$

where we have introduced the notation

$$
\sigma_{s}^{\kappa, \kappa^{\prime}}(\chi, l)=\sum_{\substack{\mathfrak{q} \in C_{\kappa}^{-1} C_{\kappa^{\prime}}^{-1} \\ \text { integral } \\ \mathfrak{q} \mid \mathfrak{b}^{\prime} \mathfrak{d a l}}} \chi(\mathfrak{q}) \mathrm{N}_{K / \mathbb{Q}}(\mathfrak{q})^{s} .
$$

To see this, note that $\mathfrak{a}^{*}=\mathfrak{a}^{-1} O^{*}$. Note also that $\mathfrak{b}^{\prime} \mathfrak{d} l$ is integral. In particular, this sum is finite. The integral equals

$$
\left\{\int_{-\infty}^{+\infty} \frac{y_{1}^{s_{1}} e^{-2 \pi i l^{(1)} x_{1}} d x_{1}}{\left(x_{1}^{2}+y_{1}^{2}\right)^{s_{1}}}\right\} \cdots\left\{\int_{\mathbb{C}} \frac{y_{n}^{s_{n}} e^{-4 \pi i \operatorname{Re}\left(l^{(n)} x_{n}\right)} d x_{n}}{\left(\left|x_{n}\right|^{2}+y_{n}^{2}\right)^{s_{n}}}\right\} .
$$

Using the well-known result that

$$
\int_{-\infty}^{+\infty} \frac{e^{-2 \pi i A t}}{\left(1+t^{2}\right)^{s}} d t=\frac{2 \pi^{s}|A|^{s-1 / 2} K_{s-1 / 2}(2 \pi|A|)}{\Gamma(s)}
$$

see for example [4, it follows that for $i \leq r_{1}$, we have

$$
\int_{-\infty}^{+\infty} \frac{y_{i}^{s_{i}} e^{-2 \pi i l^{(i)} x_{i}} d x_{i}}{\left(x_{i}^{2}+y_{i}^{2}\right)^{s_{i}}}=\frac{2 \pi^{s_{i}}\left|l^{(i)}\right|^{s_{i}-1 / 2} \sqrt{y_{i}} K_{s_{i}-1 / 2}\left(2 \pi\left|l^{(i)}\right| y_{i}\right)}{\Gamma\left(s_{i}\right)} .
$$

To compute the last $r_{2}$ factors, we apply the following integral:

$$
\int_{-\infty}^{+\infty} \int_{-\infty}^{+\infty} \frac{e^{-2 \pi i(A u-B v)}}{\left(1+u^{2}+v^{2}\right)^{s}} d u d v=\frac{2 \pi^{s}|C|^{s-1} K_{s-1}(2 \pi|C|)}{\Gamma(s)}
$$

where $A^{2}+B^{2}=C^{2}$. To prove this, make a linear change of variables and apply the integral above. Then for $i>r_{1}$, we have

$$
\int_{\mathbb{C}} \frac{y_{i}^{s_{i}} e^{-4 \pi i \operatorname{Re}\left(l^{(i)} x_{i}\right)} d x_{i}}{\left(\left|x_{i}\right|^{2}+y_{i}^{2}\right)^{s_{i}}}=\frac{(2 \pi)^{s_{i}}\left|l^{(i)}\right|^{s_{i}-1} y_{i} K_{s_{i}-1}\left(4 \pi\left|l^{(i)}\right| y_{i}\right)}{\Gamma\left(s_{i}\right)} .
$$

Now we can put all this together and obtain an expression for $a_{l}^{\kappa, \kappa^{\prime}}(y, s, m, \chi)^{*}$. The best way to state the result is that

$$
\begin{aligned}
& \hat{a}_{l}^{\kappa, \kappa^{\prime}}(y, s, m, \chi)=\frac{\chi\left(\mathfrak{a}^{\prime}\right)}{\mathrm{N}_{K / \mathbb{Q}}\left(\mathfrak{a}^{\prime}\right)^{2 s-2}} \\
& \cdot 2^{d} \mathrm{~N}_{K / \mathbb{Q}}(\mathfrak{d} l)^{s-1 / 2} \chi_{m / 2}(l)^{-1} \sigma_{1-2 s}^{\kappa, \kappa^{\prime}}(\chi, l) \sqrt{y_{1} \cdots y_{n}^{2}} K_{s-1 / 2}(y, l, m),
\end{aligned}
$$

where $\mathfrak{d}$ is the different,

$$
\chi_{m / 2}(l)=\prod_{i=1}^{n}\left|l^{(i)}\right|^{-\pi i\left\langle m, e_{i}\right\rangle}
$$

and

$$
K_{s}(y, l, m)=K_{s_{1}}\left(2 \pi\left|l^{(1)}\right| y_{1}\right) \cdots K_{s_{n}}\left(4 \pi\left|l^{(n)}\right| y_{n}\right) .
$$


To get $a_{l}^{\kappa, \kappa^{\prime}}(y, s, m, \chi)$, we arrange them in a column vector

$$
a_{l}^{\kappa^{\prime}}(y, s, m, \chi)=\left\{a_{l}^{\kappa, \kappa^{\prime}}(y, s, m, \chi)\right\},
$$

and similarly we define $\hat{a}_{l}^{\kappa^{\prime}}(y, s, m, \chi)$. We also put

$$
\sigma_{s}^{\kappa^{\prime}}(\chi, l)=\left\{\sigma_{s}^{\kappa, \kappa^{\prime}}(\chi, l)\right\} \text {. }
$$

Then what we have shown is the following

Lemma 6.1. Let $\kappa^{\prime}$ be a fixed cusp. For a nonzero $l \in \mathfrak{b}^{\prime *}$, we have

$$
a_{l}^{\kappa^{\prime}}(y, s, m, \chi)=\frac{\chi\left(\mathfrak{a}^{\prime}\right)}{\mathrm{N}_{K / \mathbb{Q}}\left(\mathfrak{a}^{\prime}\right)^{2 s-2}} a_{l}^{\kappa^{\prime}}(s, m, \chi) \sqrt{y_{1} \cdots y_{n}^{2}} K_{s-1 / 2}(y, l, m),
$$

where

$$
a_{l}^{\kappa^{\prime}}(s, m, \chi)=2^{d} \mathrm{~N}_{K / \mathbb{Q}}(\mathfrak{d} l)^{s-1 / 2} \chi_{m / 2}(l)^{-1} \hat{\Lambda}(2 s, \chi)^{-1} \sigma_{1-2 s}^{\kappa^{\prime}}(\chi, l)
$$

with notation as above.

\section{The SCATTERING MATRIX}

In the previous two sections, we have computed the Fourier expansion of the Eisenstein vector $\mathfrak{E}(z, s, m, \chi)$ around a cusp $\kappa^{\prime}$. We have

$$
\mathfrak{E}\left(\sigma^{\prime} z, s, m, \chi\right)=\sum_{l \in \mathfrak{b}^{\prime *}} a_{l}^{\kappa^{\prime}}(y, s, m, \chi) e^{2 \pi i \operatorname{Tr}_{K / \mathbb{Q}}(l x)},
$$

where the constant term is

$a_{0}^{\kappa^{\prime}}(y, s, m, \chi)=e^{\kappa^{\prime}} \frac{\mathrm{N}_{K / \mathbb{Q}}\left(\mathfrak{a}^{\prime}\right)^{s}}{\chi\left(\mathfrak{a}^{\prime}\right)} y_{1}^{s_{1}} \cdots y_{n}^{s_{n}}+\frac{\chi\left(\mathfrak{a}^{\prime}\right)}{\mathrm{N}_{K / \mathbb{Q}}\left(\mathfrak{a}^{\prime}\right)^{2 s-2}} \Phi^{\kappa^{\prime}}(s, m, \chi) y_{1}^{1-s_{1}} \cdots y_{n}^{2-s_{n}}$

where $\Phi^{\kappa^{\prime}}(s, m, \chi)$ is the $\kappa^{\prime}$-th column of

$$
\Phi(s, m, \chi)=\hat{\Lambda}(2 s, \chi)^{-1} \hat{\Lambda}(2 s-1, \chi) \mathbb{P} .
$$

For nonzero $l \in \mathfrak{b}^{\prime *}$, the Fourier coefficients are

$$
a_{l}^{\kappa^{\prime}}(y, s, m, \chi)=\frac{\chi\left(\mathfrak{a}^{\prime}\right)}{\mathrm{N}_{K / \mathbb{Q}}\left(\mathfrak{a}^{\prime}\right)^{2 s-2}} a_{l}^{\kappa^{\prime}}(s, m, \chi) \sqrt{y_{1} \cdots y_{n}^{2}} K_{s-1 / 2}(y, l, m),
$$

where

$$
a_{l}^{\kappa^{\prime}}(s, m, \chi)=2^{d} \mathrm{~N}_{K / \mathbb{Q}}(\mathfrak{d} l)^{s-1 / 2} \chi_{m / 2}(l)^{-1} \hat{\Lambda}(2 s, \chi)^{-1} \sigma_{1-2 s}^{\kappa^{\prime}}(\chi, l) .
$$

Let us put $\kappa^{\prime}=\infty$. Using the exponential decay of Macdonald's Bessel function and the meromorphic continuation of partial Hecke $L$-series, we see that this gives the meromorphic continuation of the Eisenstein series. Using the functional equation of partial Hecke $L$-series, we shall now derive the functional equation of the Eisenstein vector. First we check that

$$
\Phi(s, m, \chi) \Phi(1-s,-m, \bar{\chi})=I_{h} .
$$

This follows immediately from the relation

$$
\hat{\Lambda}(s, \chi) \mathbb{P}=\chi(\mathfrak{d}) \mathbb{P}_{\mathfrak{d}} \hat{\Lambda}(1-s, \bar{\chi}),
$$

where $\mathbb{P}_{\mathfrak{d}}$ is the permutation matrix

$$
\mathbb{P}_{\mathfrak{o}}=\left\{\delta_{\pi(\kappa), \kappa^{\prime}}\right\}
$$


and where $\pi$ is the permutation of the $\{\kappa\}$ given by

$$
C_{\pi(\kappa)}^{-1}=[\mathfrak{d}] C_{\kappa}
$$

Macdonald's Bessel functions are even in the $s$ variable, and hence

$$
K_{-s}(y, l,-m)=K_{s}(y, l, m) .
$$

The last relation we need in order to prove the functional equation is

Lemma 7.1. $\mathbb{P}_{\mathfrak{d}} \sigma_{s}^{\kappa^{\prime}}(\chi, l)=\mathrm{N}_{K / \mathbb{Q}}\left(\mathfrak{b}^{\prime} \mathfrak{d} l\right)^{s} \chi\left(\mathfrak{b}^{\prime} \mathfrak{d} l\right) \sigma_{-s}^{\kappa^{\prime}}(\bar{\chi}, l)$.

Proof. By definition of $\pi$, we see that

$$
\left(\mathbb{P}_{\mathfrak{d}} \sigma_{s}^{\kappa^{\prime}}(\chi, l)\right)_{\kappa}=\sum_{\substack{\mathfrak{q} \in[\mathfrak{d}] C_{\kappa} C_{\kappa^{\prime}}^{-1} \\ \text { integral } \\ \mathfrak{q} \mid \mathfrak{b}^{\prime} \mathfrak{d} l}} \chi(\mathfrak{q}) \mathrm{N}_{K / \mathbb{Q}}(\mathfrak{q})^{s}
$$

There is a bijection

$$
\left\{\begin{array}{c}
\mathfrak{q} \in[\mathfrak{d}] C_{\kappa} C_{\kappa^{\prime}}^{-1} \\
\text { integral } \\
\mathfrak{q} \mid \mathfrak{b}^{\prime} \mathfrak{d} l
\end{array}\right\} \approx\left\{\begin{array}{c}
\mathfrak{q} \in C_{\kappa}^{-1} C_{\kappa^{\prime}}^{-1} \\
\text { integral } \\
\mathfrak{q} \mid \mathfrak{b}^{\prime} \mathfrak{d} l
\end{array}\right\}
$$

given by $\mathfrak{q} \mapsto \mathfrak{q}^{-1} \mathfrak{b}^{\prime} \mathfrak{d} l$. Hence,

$$
\left(\mathbb{P}_{\mathfrak{d}} \sigma_{s}^{\kappa^{\prime}}(\chi, l)\right)_{\kappa}=\sum_{\substack{\mathfrak{q} \in C_{\kappa}^{-1} C_{\kappa^{\prime}}^{-1} \\ \text { integral } \\ \mathfrak{q} \mid \mathfrak{b}^{\prime} \mathfrak{d} l}} \chi\left(\mathfrak{q}^{-1} \mathfrak{b}^{\prime} \mathfrak{d} l\right) \mathrm{N}_{K / \mathbb{Q}}\left(\mathfrak{q}^{-1} \mathfrak{b}^{\prime} \mathfrak{d} l\right)^{s}
$$

and the claim follows immediately.

Now a straightforward calculation gives us the functional equation

$$
\mathfrak{E}(z, s, m, \chi)=\Phi(s, m, \chi) \mathfrak{E}(z, 1-s,-m, \bar{\chi}) .
$$

Thus we have finally identified the scattering matrix.

Theorem 7.2. The scattering matrix for the Hilbert modular group is

$$
\Phi(s, m, \chi)=\hat{\Lambda}(2 s, \chi)^{-1} \hat{\Lambda}(2 s-1, \chi) \mathbb{P},
$$

with notation as above.

\section{The SCATTERING DETERMINANT}

In this section, we compute the scattering determinant $\operatorname{det} \Phi(s, m, \chi)$ in terms of Hecke $L$-series. In order to do so, we must compute $\operatorname{det} \hat{\Lambda}(s, \chi)$. For this purpose, we have the following well-known result:

Proposition 8.1. Let $G$ be a finite abelian group, and $f$ any function. Then

$$
\operatorname{det}_{a, b} f\left(a^{-1} b\right)=\prod_{\psi \in G^{*}} \sum_{a \in G} \psi(a) f(a),
$$

where $G^{*}=\{\psi\}$ is the group of characters. 
Proof. Let $X(G)$ denote the $|G|$-dimensional complex vector space of functions on $G$. It has two canonical bases:

$$
\text { (1) the characters }\left\{\psi: \psi \in G^{*}\right\} \text {, }
$$

(2) the functions $\left\{\delta_{b}: b \in G\right\}$ where $\delta_{b}(x)=\delta_{x, b}$.

For each $a \in G$, we have the linear map $T_{a}$ on $X(G)$ defined by $T_{a} f(x)=f(a x)$. We weight all these $T_{a}$ using $f$ and consider the linear map

$$
T=\sum_{a \in G} f\left(a^{-1}\right) T_{a}
$$

To derive the Dedekind determinant relation, we compute $\operatorname{det} T$ using the two canonical bases. It is clear that

$$
T \psi=\left\{\sum_{a \in G} \psi(a) f\left(a^{-1}\right)\right\} \psi,
$$

for every $\psi \in G^{*}$. Hence the first basis is a basis of eigenvectors, so that

$$
\operatorname{det} T=\prod_{\psi \in G^{*}} \sum_{a \in G} \psi(a) f\left(a^{-1}\right)=\prod_{\psi \in G^{*}} \sum_{a \in G} \psi(a) f(a) .
$$

On the other hand, a straightforward computation shows that

$$
T \delta_{b}=\sum_{a \in G} f\left(a^{-1} b\right) \delta_{a} .
$$

Thus we also have

$$
\operatorname{det} T=\operatorname{det}_{a, b} f\left(a^{-1} b\right) .
$$

Comparing these two expressions for $\operatorname{det} T$ finishes the proof.

Using this proposition, we can now proceed with our calculation. It follows that

$$
\operatorname{det} \hat{\Lambda}(s, \chi)=\prod_{\psi \in \mathrm{Cl}^{*}} \Lambda(s, \psi \otimes \chi) .
$$

As $\psi$ varies over $\mathrm{Cl}^{*}$, the product $\psi \otimes \chi$ varies over all Grössencharakters modulo $\mathfrak{m}=O$ extending $\chi_{m}$. We thus have

Corollary 8.2. The scattering determinant for the Hilbert modular group is

$$
\operatorname{det} \Phi(s, m, \chi)=\operatorname{sign}(\mathrm{Cl}) \prod_{\psi} \frac{\Lambda(2 s-1, \psi)}{\Lambda(2 s, \psi)},
$$

where $\psi$ varies over all Grössencharakters modulo $\mathfrak{m}=O$ extending $\chi_{m}$.

As we see, $\operatorname{det} \Phi(s, m, \chi)$ does not depend on $\chi$; so we use the notation

$$
\phi(s, m)=\operatorname{det} \Phi(s, m, \chi) .
$$

In the corollary, $\operatorname{sign}(\mathrm{Cl})$ denotes the sign of the inversion map on $\mathrm{Cl}$. This is given by

$$
\operatorname{sign}(\mathrm{Cl})=(-1)^{(h-h[2]) / 2},
$$

where $h[2]=\sharp \mathrm{Cl}[2]$. In the case where $K$ is an imaginary quadratic field (as in [2]), the 2 -torsion $h[2]$ can be expressed via genus theory as

$$
h[2]=2^{t-1} \text {, where } t \text { is the number of primes dividing } D \text {. }
$$


See for example 3]. We now consider the particularly interesting case where $m=0$. Then

$$
\phi(s, 0)=\operatorname{sign}(\mathrm{Cl}) \prod_{\psi \in \mathrm{Cl}^{*}} \frac{\Lambda(2 s-1, \psi)}{\Lambda(2 s, \psi)},
$$

and via class field theory, we can interpret this in terms of the Hilbert class field

$$
H=\text { the maximal unramified abelian extension of } K \text {. }
$$

Here unramified means everywhere unramified, also at the infinite places (so real places stay real). The Artin map induces an isomorphism

$$
r: \mathrm{Cl} \approx \operatorname{Gal}(H / K)
$$

mapping $\mathfrak{p} \mapsto \operatorname{Fr}_{\mathfrak{p}}$. It is known that the Artin $L$-function

$$
\Lambda\left(s, \psi \circ r^{-1}, H / K\right)=\Lambda(s, \psi) .
$$

See for example [5]. Then the Artin factorization formula gives

$$
\xi_{H}(s)=\prod_{\psi \in \mathrm{Cl}^{*}} \Lambda(s, \psi),
$$

where $\xi_{H}(s)$ is the Dedekind $\xi$-function of $H$. Recall that for a number field $K$, we have

$$
\xi_{K}(s)=2^{r_{2}(1-s)} \pi^{-d s / 2}|D|^{s / 2} \Gamma(s / 2)^{r_{1}} \Gamma(s)^{r_{2}} \zeta_{K}(s) .
$$

This satisfies the functional equation $\xi_{K}(1-s)=\xi_{K}(s)$. Since $H$ is everywhere unramified, real places stay real; so the Dedekind $\xi$-function of $H$ is then given by

$$
\xi_{H}(s)=2^{h r_{2}(1-s)} \pi^{-h d s / 2}\left|D_{H}\right|^{s / 2} \Gamma(s / 2)^{h r_{1}} \Gamma(s)^{h r_{2}} \zeta_{H}(s) .
$$

Then what we have shown is the following

Corollary 8.3. For $m=0$, the scattering determinant is given by

$$
\phi(s, 0)=\operatorname{sign}(\mathrm{Cl}) \frac{\xi_{H}(2 s-1)}{\xi_{H}(2 s)},
$$

with notation as above.

Using this corollary, we can give the following analytic interpretation of $\operatorname{sign}(\mathrm{Cl})$ in terms of the central critical value of $\phi(s, 0)$ :

Corollary 8.4. $\phi(1 / 2,0)=-\operatorname{sign}(\mathrm{Cl})$.

Proof. It is a fact that the only poles of $\xi_{H}(s)$ are $s=0$ and $s=1$. They are known to be simple, and their residues are given as follows:

$$
\operatorname{Res}_{s=0} \xi_{H}(s)=-\frac{2^{h r_{1}} h_{H} R_{H}}{w_{H}} \text { and } \operatorname{Res}_{s=1} \xi_{H}(s)=+\frac{2^{h r_{1}} h_{H} R_{H}}{w_{H}} .
$$

For the proof of these facts, see [5. Then we have

$$
\phi(s / 2,0)=\operatorname{sign}(\mathrm{Cl}) \frac{(s-1) \xi_{H}(s-1)}{(s-1) \xi_{H}(s)} \rightarrow-\operatorname{sign}(\mathrm{Cl}),
$$

as $s \rightarrow 1$. Hence $\phi(1 / 2,0)=-\operatorname{sign}(\mathrm{Cl})$ as we claimed. 


\section{The SCATTERING TRACE}

In this section, we compute the scattering trace $\operatorname{tr} \Phi(s, m, \chi)$ in terms of Hecke $L$-series. To compute the trace of

$$
\Phi(s, m, \chi)=\hat{\Lambda}(2 s, \chi)^{-1} \hat{\Lambda}(2 s-1, \chi) \mathbb{P},
$$

we diagonalize $\hat{\Lambda}(s, \chi)$. From the proof of the Dedekind determinant, we get that

$$
\hat{\Lambda}(s, \chi)=M^{-1} \operatorname{diag}(\ldots, \Lambda(s, \psi \otimes \chi), \ldots) M,
$$

where $M$ is the unitary matrix given by the character table

$$
M=\left\{\frac{1}{\sqrt{h}} \bar{\psi}\left(C_{\kappa}\right)\right\} .
$$

It follows from this that we may write the scattering matrix as

$$
\Phi(s, m, \chi)=M^{-1} \operatorname{diag}\left(\ldots, \frac{\Lambda(2 s-1, \psi \otimes \chi)}{\Lambda(2 s, \psi \otimes \chi)}, \ldots\right) \mathbb{P}^{M} M,
$$

where $\mathbb{P}^{M}=M \mathbb{P} M^{-1}$. Using the fact that $M$ is unitary and the orthogonality relations, we see that $\mathbb{P}^{M}$ is the permutation matrix corresponding to inversion on $\mathrm{Cl}^{*}$. From this the following corollary follows immediately.

Corollary 9.1. The scattering trace for the Hilbert modular group is

$$
\operatorname{tr} \Phi(s, m, \chi)=\sum_{\psi \text { real }} \frac{\Lambda(2 s-1, \psi \otimes \chi)}{\Lambda(2 s, \psi \otimes \chi)},
$$

where $\psi$ varies over all real characters of $\mathrm{Cl}$.

We want to compute the central scattering trace $\operatorname{tr} \Phi(1 / 2,0, \chi)$, the main motivation being that (at least for totally real fields) it occurs in the trace formula. See [1]. From the functional equation, it follows that for real $\chi$ we have

$$
\operatorname{tr} \Phi(1 / 2,0, \chi)=\sum_{\psi \text { real }}(-1)^{m_{\psi}} \psi(\mathfrak{d}),
$$

where $m_{\psi}$ is the order of vanishing of $\Lambda(s, \psi)$ at $s=0$. By the work of Hecke and Tate we know that $\Lambda(s, \psi)$ is entire if $\psi \neq 1$. By the Artin factorization formula and the computation of residues for Dedekind $\xi$-functions, we thus see that $m_{\psi}=0$ if $\psi \neq 1$. If on the other hand $\psi=1$, we know that $m_{\psi}=-1$. Using the orthogonality relations for the group $\mathrm{Cl} / \mathrm{Cl}^{2}$, it follows that

$$
\operatorname{tr} \Phi(1 / 2,0, \chi)=h[2]-2,
$$

when $[\mathfrak{d}] \in \mathrm{Cl}^{2}$. It is a result of Hecke that this condition is always satisfied. Thus we have

Corollary 9.2. For real $\chi$ the central scattering trace is given by

$$
\operatorname{tr} \Phi(1 / 2,0, \chi)=h[2]-2,
$$

with notation as above.

When $K$ is quadratic imaginary, we can rewrite this via genus theory and obtain the expression in [2]. We note that we can easily derive the formula

$$
\phi(1 / 2,0)=-\operatorname{sign}(\mathrm{Cl})
$$


from the above. Let $A=\Phi(1 / 2,0, \chi)$. Then $A^{2}=I_{h}$ and hence it has eigenvalues \pm 1 . Let $m_{ \pm}$be the multiplicities. Then we have

$$
m_{+}+m_{-}=h \text { and } m_{+}-m_{-}=h[2]-2 .
$$

From this it follows that

$$
m_{+}=\frac{1}{2}(h+h[2]-2) \quad \text { and } \quad m_{-}=\frac{1}{2}(h-h[2]+2),
$$

and we see that $\operatorname{det} A=(-1)^{m_{-}}=-\operatorname{sign}(\mathrm{Cl})$.

\section{REFERENCES}

1. Efrat, Isaac Y., The Selberg trace formula for $\mathrm{PSL}_{2}(\mathbf{R})^{n}$, Mem. Amer. Math. Soc. 65 (1987), no. 359, iv+111 pp. MR 88e:11041

2. Efrat, I. and Sarnak, P., The determinant of the Eisenstein matrix and Hilbert class fields, Trans. Amer. Math. Soc. 290 (1985), no. 2, 815-824. MR 87b:11039

3. Janusz, Gerald J., Algebraic number fields, Pure and Applied Mathematics, Vol. 55. Academic Press [A Subsidiary of Harcourt Brace Jovanovich, Publishers], New York-London, 1973. x+220 pp. MR 51:3110

4. Kubota, Tomio, Elementary theory of Eisenstein series, Kodansha Ltd., Tokyo; Halsted Press [John Wiley and Sons], New York-London-Sydney, 1973. xi+110 pp. MR 55:2759

5. Neukirch, Jürgen, Algebraic number theory, Translated from the 1992 German original and with a note by Norbert Schappacher. With a foreword by G. Harder. Grundlehren der Mathematischen Wissenschaften [Fundamental Principles of Mathematical Sciences], 322. Springer-Verlag, Berlin, 1999. xviii+571 pp. MR 2000m:11104

6. Selberg, A., Harmonic analysis and discontinuous groups in weakly symmetric Riemannian spaces with applications to Dirichlet series, J. Indian Math. Soc. (N.S.) 20 (1956), 47-87. MR 19:531g

7. Selberg, Atle, Recent developments in the theory of discontinuous groups of motions of symmetric spaces, 1970 Proceedings of the Fifteenth Scandinavian Congress (Oslo, 1968), Lecture Notes in Mathematics, Vol. 118 Springer, Berlin, pp. 99-120 MR 41:8595

Department of Mathematics, Ny Munkegade, 8000 Aarhus C, Denmark

E-mail address: mazanti@imf .aau.dk

Current address: Department of Mathematics, California Institute of Technology, Pasadena, California 91125, USA 